\title{
Gradhiva
}

GRADHIV

Revue d'anthropologie et d'histoire des arts

$25 \mid 2017$

Gottfried Semper, habiter la couleur

\section{L'ornement à la conquête de soi. Tectonique, métaphysique et anthropologie chez Karl Bötticher et Gottfried Semper}

Ornament conquering itself. Tectonic, metaphysics, and anthropology in the works of Karl Bötticher and Gottfried Semper

\section{Rémi Labrusse}

\section{OpenEdition}

Journals

Édition électronique

URL : http://journals.openedition.org/gradhiva/3363

DOI : 10.4000/gradhiva.3363

ISSN : 1760-849X

\section{Éditeur}

Musée du quai Branly Jacques Chirac

Édition imprimée

Date de publication : 31 mai 2017

Pagination : $50-79$

ISBN : 978-2-35744-095-1

ISSN : 0764-8928

\section{Référence électronique}

Rémi Labrusse, «L'ornement à la conquête de soi. Tectonique, métaphysique et anthropologie chez Karl Bötticher et Gottfried Semper », Gradhiva [En ligne], 25 | 2017, mis en ligne le 31 mai 2019, consulté le 04 janvier 2020. URL : http://journals.openedition.org/gradhiva/3363 ; DOI : 10.4000/ gradhiva.3363 



\section{L'ornement à la conquête de soi}

Tectonique, métaphysique et anthropologie chez Karl Bötticher et Gottfried Semper

par Rémi Labrusse

La «tectonique» selon Karl Bötticher (1806-1889) peut être considérée comme une philosophie première de la construction, fondée sur l'idée de grammaire ornementale. Elle aboutit à une définition véritablement révolutionnaire de l'ornement comme allégorie de la construction, c'est-à-dire de soi-même, suivant une loi de réflexivité infinie. À partir de l'analyse de cette dimension auto-allégorique, il s'agit de comprendre comment une métaphysique de la vérité constructive, chez Bötticher, s'oppose à une anthropologie du désir de fictions spatiales, chez Semper. 
1. Les passages ci-après, consacrés à la carrière et à la pensée de Karl Bötticher, constituent une version remaniée et augmentée de l'article de l'auteur, «Référence hellénique et ontologie de l'ornement. La "tectonique" de Karl Bötticher ", in Espagne et Maufroy (dir.) 2016: 223-246.

2. Sauf mention contraire, les éléments biographiques sont tirés de ce texte, qui synthétise les nécrologies et biographies antérieures. Voir également Streiter 1896: 5-9.
Depuis une vingtaine d'années, la comparaison entre les pensées de Karl Bötticher et de Gottfried Semper constitue une sorte de passage obligé de toutes les études sur la notion de tectonique et, à partir de là, sur l'histoire des théories architecturales au XIX ${ }^{\mathrm{e}}$ siècle. Elle est généralement fondée sur la mise en rapport entre Die Tektonik der Hellenen («La Tectonique des Hellènes ") de Bötticher, dont les premières formulations datent de 1840, et Der Stil in den technischen und tektonischen Künsten («Le Style dans les arts techniques et tectoniques") de Semper, dont le premier volume est paru vingt ans plus tard. Elle aboutit le plus souvent - et à juste titre - à mettre en lumière leurs divergences, entre idéalisme (chez Bötticher) et matérialisme (chez Semper). II s'agit ici de reprendre la comparaison pour montrer qu'on ne saurait exagérer ni la proximité ni l'opposition entre les deux pensées. L'objectif n'est pas de revaloriser un précurseur méconnu, encore que ce que Semper a puisé chez Bötticher est sans doute sous-évalué, mais de montrer que ces deux conceptions constituent deux propositions exemplaires articulant pensée de la construction et vision du monde, à l'ère industrielle. On fera apparaître que ces deux efforts à bien des égards antithétiques se sont fondés sur une redéfinition du concept d'ornement, considéré en tant que moteur intellectuel de celui de tectonique, et que le premier y a donc acquis à la fois un prestige et une instabilité conceptuelle extrêmes, qui continuent aujourd'hui de l'affecter, au-delà des seules théories de l'architecture.

\section{Dessin d'ornement, archéologie, philosophie}

Pour ce faire, il convient de revenir d'abord sur l'élaboration de la pensée de Bötticher, sur son contexte et, au fond, sur son étrangeté ${ }^{1}$. En amont de l'œuvre, ce qui confère au personnage sa singularité, c'est son appartenance, tout au long de sa carrière, à des sphères intellectuelles et professionnelles d'ordinaire séparées: la pratique du dessin d'ornementation, l'érudition archéologique et la spéculation philosophique.

D’origine modeste, formé au départ pour devenir contremaître dans la petite ville de Nordhausen, en Thuringe, Bötticher s'est lancé en autodidacte dans la pratique ornementale (Michaelis $1903^{2}$ ). Grâce à des talents exceptionnels de dessinateur, il a pu monter à Berlin en 1827 et s'assurer un début de notoriété en collaborant aux efforts contemporains de la Prusse pour réformer la pédagogie et la pratique du dessin ornemental, dans les années 1830, avec le soutien de l'architecte Karl Friedrich Schinkel et du conseiller d'État Christian Peter Wilhelm Friedrich Beuth. Ce dernier était directeur depuis 1819 de la Députation technique pour l'industrie (Technische Deputation für Gewerbe), l'administration responsable de la stratégie industrielle du royaume. À ce titre, il avait lancé à partir de 1821, en lien étroit avec Schinkel, le grand projet éditorial des Vorbilder für Fabrikanten und Handwerker («Modèles pour fabricants et artisans») [Beuth 1821-1837], auquel Bötticher a participé in extremis, en dessinant les dernières planches du recueil en 1837. Dans l'ensemble de cette publication monumentale, plusieurs éléments fondateurs de la pensée postérieure de Bötticher se manifestent déjà, sans être pour autant théorisés: l'unité «tectonique» (même si le mot n'existe pas encore) entre architecture et objets mobiliers (l'ouvrage est divisé en trois sections, respectivement consacrées aux «ornementations 
architecturales et autres », aux «ustensiles, récipients et petits monuments » et aux «étoffes et tissages»); l'importance spécifique du textile; une approche rationaliste de la Grèce antique, mesures géométriques à l'appui (fig. 2), avec comme objectif central, écrit Beuth, «la mise en application d'un goût classique dans nos productions industrielles » (Beuth 1821-1837 II: v); enfin, l'alliance entre philologie et science de l'ornement, ajoutant à l'apprentissage des langues anciennes celui d'une langue ornementale sévèrement normée (ibid.: ix ${ }^{3}$ ), pour assurer au pays une cohésion politico-esthétique et pour lutter contre la montée à travers l'Europe d'un éclectisme «dépassant la barbarie des derniers jours de l'Empire romain » (ibid. : viii), dont on craignait l'effet socialement délétère.

Dans ses propres planches, à vrai dire, Bötticher se démarque de la tonalité essentiellement antiquisante du reste des Modèles par l'origine des objets représentés, puisqu'il s'agit de fragments textiles conservés dans des églises protestantes du centre et du nord de l'Allemagne (fig. 4). Cette rupture est visuellement accentuée par le recours à la polychromie, grâce à l'invention récente de la lithographie en couleurs. Mais il ne s'agit en rien d'un basculement dans le pittoresque médiéval. Au contraire, l'intention est toujours de délivrer un message rationaliste global, en vidant de son contenu la vieille querelle prussienne opposant les partisans du modèle grec et ceux du modèle gothique. Ce n'est pas le Moyen Âge comme tel qui est mis en avant, c'est un monde musical d'arabesques et de formes géométriques planes, strictes compositions de lignes et de couleurs sans matière, sans histoire et sans lieu, situant l'ornement en amont du regard historique (qui l'aurait assigné à une période et à un lieu donnés) aussi bien que du regard technique (qui l'aurait fait dépendre de tel ou tel type de production).

Loin d'être opposée à la pensée industrielle, cette abstraction graphique en constitue le soubassement nécessaire, faisant prévaloir le dessein ${ }^{4}$, le dispositif conceptuel, la visée de l'esprit, sur les hésitations de la main et le travail artisanal de la matière. Du reste, le jeune Bötticher s'est aussi passionnément employé, à la même époque, à développer des applications industrielles. Il a appris personnellement le tissage en 1834, en s'engageant comme apprenti dans la manufacture de soie Gropius, et a déposé des brevets d'invention, notamment celui d'un nouveau siège pour les métiers à tisser Jacquard, lequel, acheté par l'État prussien, a contribué à améliorer la compétitivité des textiles nationaux. C'est au nom de ce double intérêt pour le dessin ornemental et pour les schémas techniques de production qu'il a finalement abordé l'architecture et est devenu membre de l'Union des architectes berlinois (Architekturverein) en 1833 (même s'il n'a pas réalisé lui-même de construction, s'en tenant à quelques rares opérations de décoration intérieure).

Tout en évoluant alors vers la réflexion théorique et vers le savoir archéologique, Bötticher n'a jamais délaissé sa première pratique de dessinateur industriel. Dans les années 1830 et 1840, il a multiplié les ouvrages de planches d'ornement ${ }^{5}$; il a également enseigné le dessin d'ornement à la Manufacture royale de porcelaine, puis a dirigé l'école de dessin (Dessinateurschule) qui venait d'être créée au sein de l'Institut d'industrie (Gewerbeinstitut); et surtout, à partir de 1844, il a exercé en tant que professeur
3. "La diffusion en tous lieux des meilleurs [modèles], inaccessibles autrement, leur imitation et la diffusion de ces imitations parmi les élèves, quand bien même ils n'y réussiraient pas aussi bien, devaient agir sur l'éducation artistique classique de la nation avec des résultats autrement plus heureux que ne l'aurait permis la seule étude des langues anciennes. "Toutes les citations sont traduites de l'allemand par l'auteur.

4. En Angleterre, au même moment, le terme de "design" commence à être employé dans cette acception : voir Varela Braga 2014.

5. Voir Ornamentenbuch zum praktischen

Gebrauche für Architekten, Decorations- und

Stubenmaler, TapetenFabrikanten, usw., 18341844 ( $1^{\text {re }}$ et $2^{\mathrm{e}}$ livraisons : $3^{\mathrm{e}}$ livraison:

Ornamentenbuch zum praktischen Gebrauche für Möbeltischler); Die Holzarchitektur des Mittelalters, mit Anschluss der schönsten in dieser Epoche entwickelten Produkte der gewerblichen Industrie, 1835-1841; Die Ornamenten-Schule, 1838; Die Dessinateurschule, 1839; Ornament-Vorbilder, 1858. 


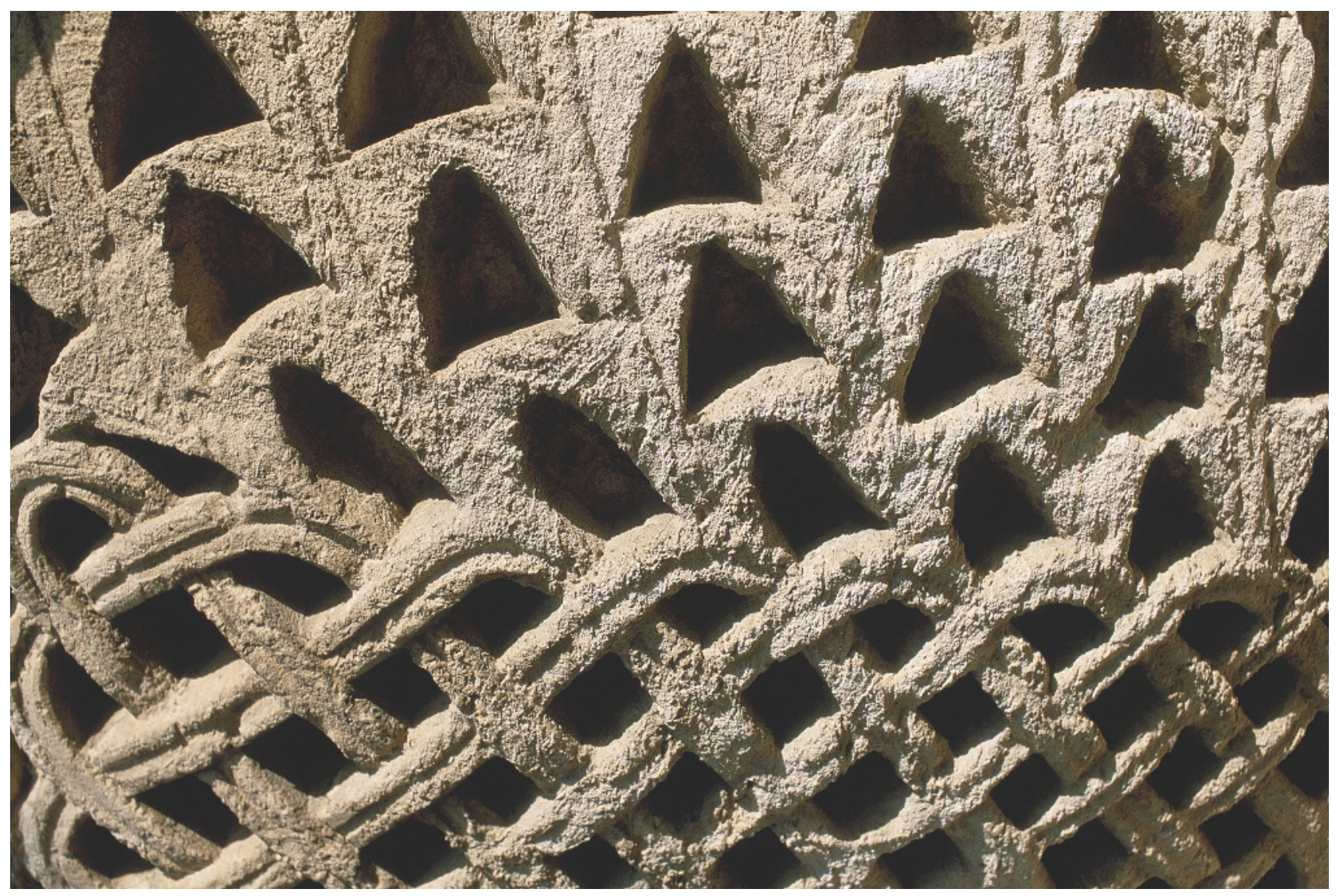

fig. 1

Détail d'un chapiteau copte.

Égypte, ves. ap. J.-C.

Port Saïd, Musée National.

Photo Hervé Champollion/

akg-images.

ci-contre

fig. 2

Karl Bötticher, «La

construction des membres architectoniques, rendue visible par les indications auxiliaires ", in Christian Peter Wilhelm Friedrich Beuth (dir.), Modèles pour fabricants et artisans.

Berlin, Petsch, 1821-1830, partie 1, section 1, feuille 1a Allemagne, Berlin,

Kupferstichkabinett (SMPK). Photo (C) BPK,

Berlin, Dist. RMN-Grand

Palais / image BPK. 

6. «Sa grandeur était incontestée, en tant qu'artiste créateur, dans son domaine spécifique, l'ornementation. [...] En tant que dessinateur, il s'est approché plus qu'aucun moderne du sentiment artistique des Grecs. [...] Et nous autres, ses jeunes contemporains berlinois, il faut bien dire que nous avons tous un peu

"böttiché" à l'époque. de tectonique à la prestigieuse Académie d'architecture - ou de construction - (Bauakademie) fondée en 1799 à Berlin - institution dans laquelle il allait enseigner pendant trente-six ans. Là, ses compétences en dessin ornemental ont contribué à sa renommée auprès de plusieurs générations d'élèves ingénieurs et architectes (Ebe 1890: 5536) aux yeux desquels il était parvenu à incarner, plus qu'aucun autre, le sacre intellectuel et savant du dessinateur d'ornements dans l'Allemagne du XIX siècle.

Le second versant de la vie intellectuelle de Bötticher est constitué par ses études archéologiques et historiques sur la Grèce archaïque et classique. Au prix d'un travail démesuré, le petit élève contremaître de Nordhausen est finalement devenu une autorité majeure - quoique toujours contestée - dans le domaine des études helléniques, qu'il s'agisse d'architecture, d'iconographie religieuse, d'anthropologie du sacré ou de muséologie (il a succédé à Eduard Gerhard en tant que directeur du département des sculptures antiques du musée de Berlin de 1868 à 1875). En ouvrant ainsi les frontières entre création artistique néoclassique et sciences de l'Antiquité, il pouvait légitimement s'enorgueillir d'avoir dépassé l'œuvre de son maître Schinkel, dont les connaissances antiquaires n'avaient pas donné lieu, de son vivant, à des publications significatives, par contraste avec son extrême productivité artistique.

De l'esprit sévèrement idéaliste des Modèles - y compris ses propres planches d'ornementation -, Bötticher a gardé la conviction que toute pensée de l'art et de l'architecture grecs était en fait une pensée du rationalisme constructif et ornemental en son essence la plus pure. Cette architecture "que nous désignons sous le terme d'hellénique», écrit-il, «n'est en réalité que le fruit mûr, le résultat épuré de toute architecture» (Bötticher 1846: 112). En attestent, dans les mêmes années, les simplifications abstraites des planches de sa Tectonique des Hellènes (fig. 5 et 6), qu'il a bien sûr dessinées lui-même et qui s'éloignent de la Grèce réelle pour rejoindre un monde de schémas graphiques décontextualisés, propres à «déduire de l'art national grec les lignes fondamentales d'un art absolu », comme l'écrit son élève Gustav Ebe (1890: 553). Le premier voyage en Grèce de Bötticher ne date d'ailleurs que de 1862, avec l'archéologue Ernst Curtius et l'architecte Heinrich Strack, et le second de 1877-1878, à l'occasion duquel il a rencontré pour la première fois, par hasard, Gottfried Semper, dans un hôtel de Venise (Michaelis 1903: 153). La Grèce de Bötticher fait donc partie intégrante d'un monde fondamentalement allemand et même spécifiquement prussien, où un rêve néohellénique a sous-tendu l'ambition de construire la nouvelle nation germanique. Cet état d'esprit politico-esthétique, maintes fois étudié (Espagne et Maufroy [dir.] 2016), a conduit à faire de la Grèce antique une figure de l'universel, tout en incarnant cet universel dans le temps historique de la nation allemande en formation: c'est à elle, pensait-on - avec Berlin, «Athènes au bord de la Spree» (Espagne 2016: 98), pour capitale -, que revenait la mission destinale de rendre l'idéalité grecque à nouveau présente et efficiente pour le reste de l'Europe moderne. Sur cet horizon grécogermanique a surgi la figure de l'Indo-Européen (Demoule 2014: 59 sq.) et, plus particulièrement, du Dorien, maître autochtone de sa propre culture, dont l'historien et archéologue Karl Otfried Müller, produit de l'université berlinoise et lui-même professeur à Göttigen, s'est donné pour mission 
d'affirmer la suprématie (Müller 1824), contre la théorie de son concurrent Ludwig Ross sur «l'Orient » comme «berceau » (Wiege) de la culture grecque (Fittschen 2005).

\section{La tectonique: invention d'un concept}

C'est dans ce contexte que Bötticher a élaboré son concept de «tectonique». Sur un plan purement sémantique, il est redevable du mot à Müller, qui l'emploie dans son Handbuch der Archaelogie der Kunst («Manuel d'archéologie artistique»), premier véritable exposé d'archéologie grecque en Europe, en 1830. C'est d'ailleurs à Müller, «le chercheur», en même temps qu'à Schinkel, le «précurseur», qu'il dédie La Tectonique des Hellènes, en 1844. Au-delà de la thèse racialiste - qu'il endosse - sur l'autonomie de la culture dorienne des «Hellènes », Bötticher doit à l'archéologue l'insistance sur la dimension technique de la science archéologique, dont la «tectonique» constitue l'horizon conceptuel:

Indéniablement, la forme géométrique peut être conçue conformément à des lois artistiques et devenir une forme artistique. Toutefois, ce genre de formes artistiques ne semble presque jamais autonome, pour des raisons qui tiennent au rapport qu'entretient l'art avec le reste de la vie des hommes et des peuples. Au contraire, elles sont attachées à une création qui remplit une finalité et répond à certains besoins vitaux. De là vient une série d'arts par lesquels sont configurés et façonnés des ustensiles, des récipients, des habitations et des lieux de rassemblement public en fonction de leur destination, bien sûr, mais aussi en accord avec des émotions et des idées artistiques. Nous appelons cette série d'activités hybrides la tectonique ${ }^{7}$. À son sommet se place l'architectonique, qui est la plus capable de s'élever au-dessus du besoin et d'exposer puissamment des impressions profondes. (Müller 1830: 10)

Ce faisant, Müller pose en principe de méthode que la compétence technologique est indispensable à une approche rigoureuse des formes dans I'histoire. Autrement dit, son livre promettait à Bötticher que son propre statut de technicien, soucieux des questions industrielles autant que du graphisme, loin d'être un obstacle socioprofessionnel à l'affirmation d'un savoir archéologique, en était la suprême garantie. Ainsi furent rassemblées, autour de 1840, les conditions de possibilité intellectuelles et psychologiques d'un concept, la «tectonique», par l'entremise duquel Bötticher a utilisé des pratiques professionnelles aussi diverses que l'archéologie, la philologie grecque, les techniques constructives et le dessin d'ornementation comme tremplins pour déployer une pensée de l'autoréflexivité ornementale. On aura bientôt l'occasion de constater à quel point cette union entre tectonique et ornement a pu conférer à ce dernier un prestige inouï, en l'articulant simultanément à l'intelligence pratique du technicien et à la conceptualité pure de l'architecte. Réciproquement, le concept de tectonique allait en tirer sa propre aura, s'enracinant rapidement dans les langues allemande puis française et anglaise pour désigner toutes les approches du réel en tant que système de structures rationnelles. À la fin du siècle, il finira par trouver son site d'élection le plus populaire dans le domaine de la géologie,
7. Je souligne. 


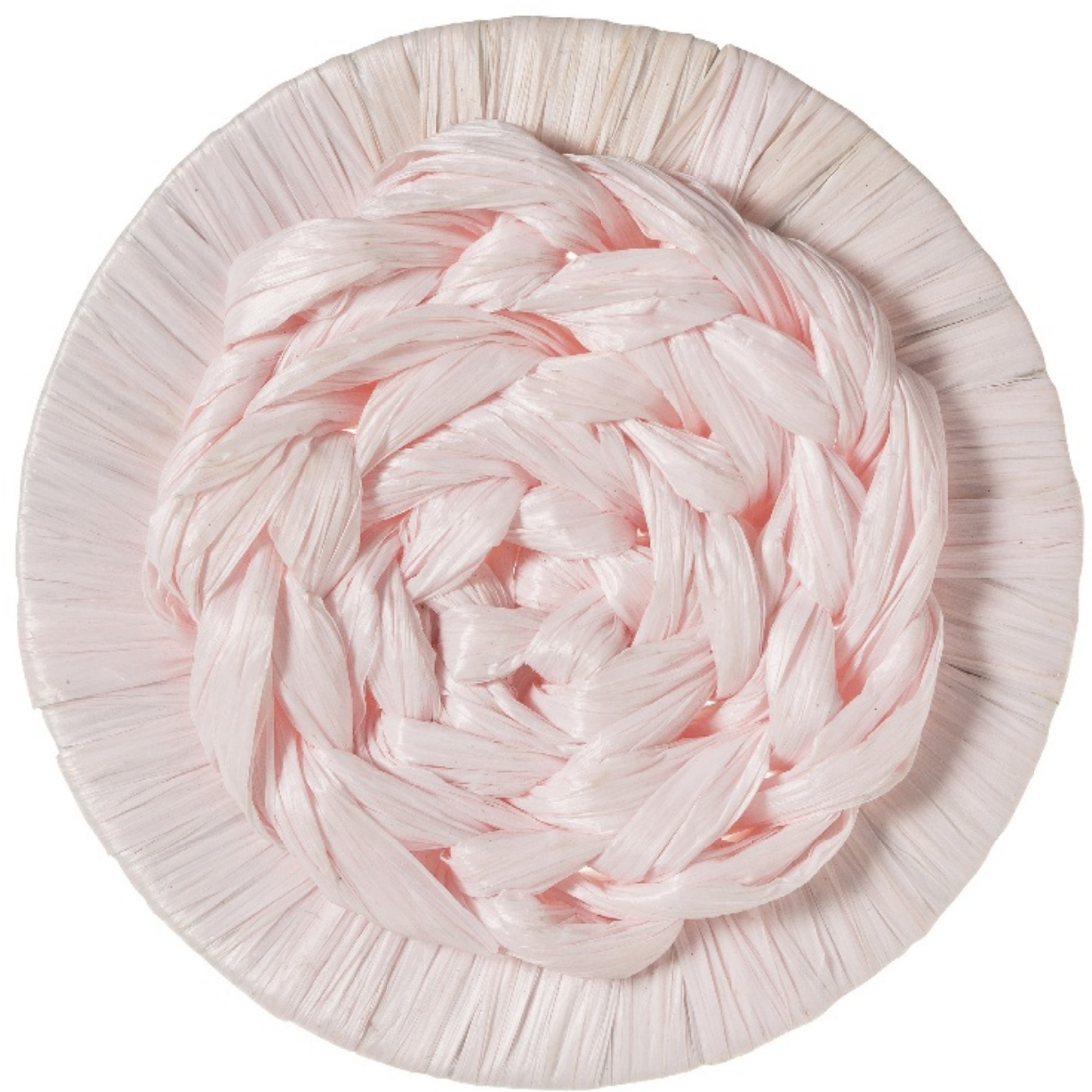

$\mathrm{fig}=3$

Bouton. France. 1950-1960, anneau métallique,

tressage de raphia artificiel, diamètre $4,1 \mathrm{~cm}$. Paris,

Les Arts Décoratifs, anc. coll. Loïc Allio. Photo Les

Arts Décoratifs, Paris/Jean Tholance/akg-images.

\section{ci-contre}

fig. 4

Karl Bötticher, « Motifs de diverses broderies en provenance d'Eisleben », in Christian Peter Wilhelm Friedrich Beuth (dir.), Modèles pour fabricants et artisans. Berlin, Petsch, 1831-1837, partie 2 , section 3, feuille 17 . 38.18-1991. Allemagne, Berlin, Kupferstichkabinett (SMPK). Photo @ BPK,

Berlin, Dist. RMN-Grand Palais/image BPK. 

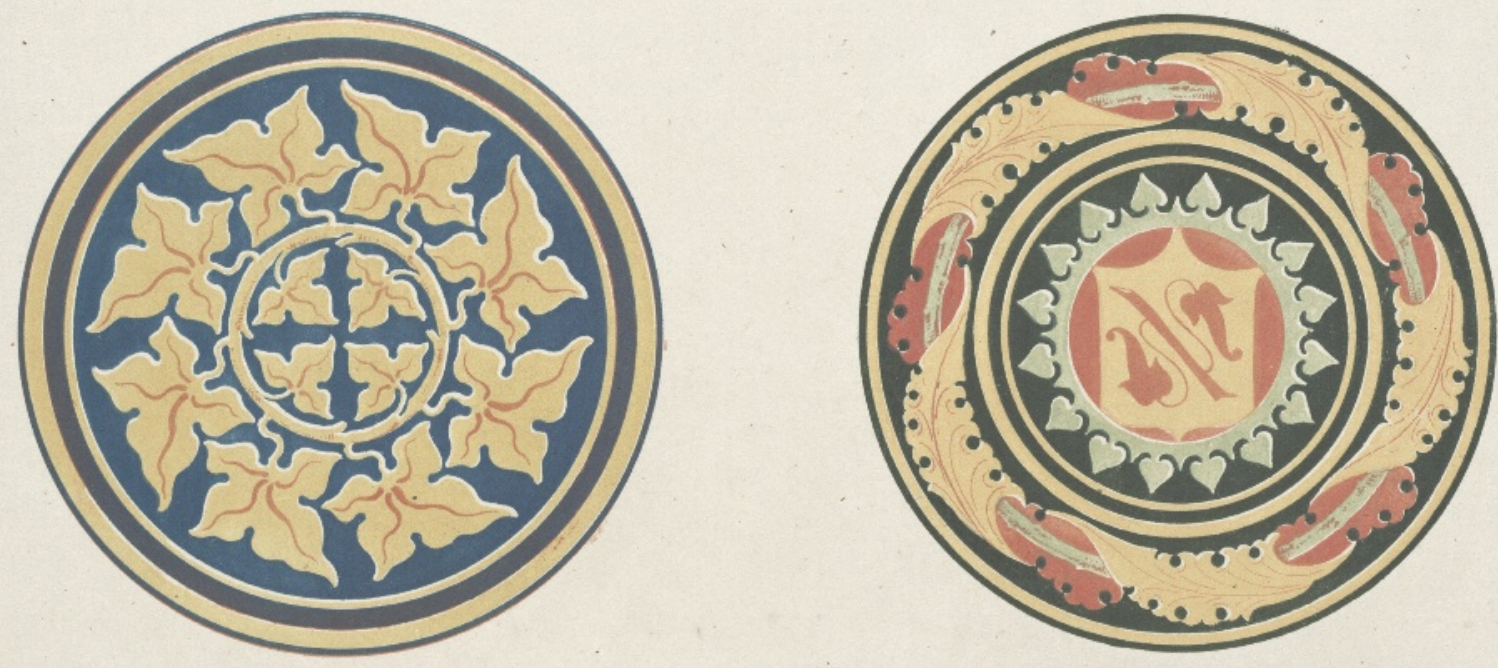

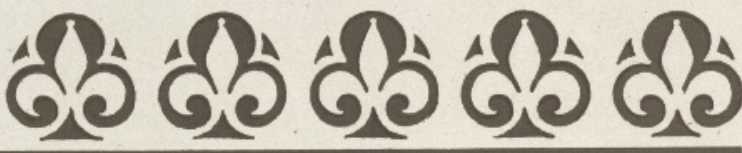
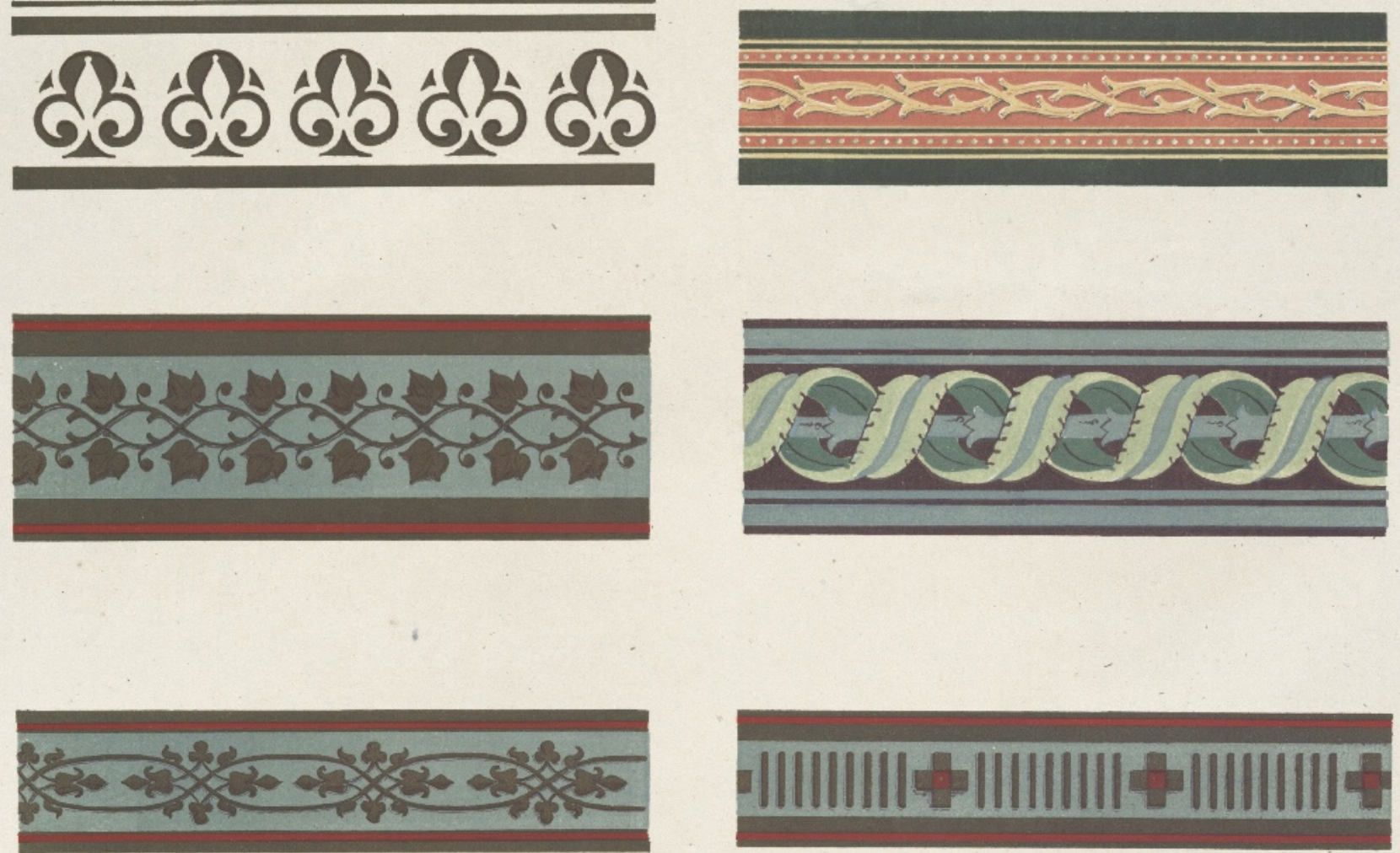
8. Sur le contexte intellectuel d'émergence de cet ouvrage fondateur. voir notamment Mayer 2004

9. Rappelons qu'en 1879 les académies d'architecture et d'industrie de Berlin ont fusionné en une unique École technique supérieure (Technische Hochschule).
Iorsqu'on décrira la planète comme un ensemble de plaques articulées les unes aux autres (jusqu'à la formulation complète de la théorie de la dérive des continents et de la «tectonique» des plaques par le géologue et climatologue Alfred Wegener au début des années 1910). Autrement dit, le rapide et vaste succès de cette vision architecturalisée du monde, symptôme de l'irrésistible montée en puissance d'un objectivisme constructiviste et d'un imaginaire mécaniste dans les consciences occidentales modernes, a pris corps grâce à un mot forgé, au départ, à l'intersection de l'archéologie classique et de la théorie de l'ornement industriel.

La philosophie tectonique de Bötticher, quant à elle, s'est exprimée pour la première fois dans le journal de l'architecte Ludwig Förster à Vienne, l'Allgemeine Bauzeitung, sous le titre «Développement des formes de la tectonique hellénique» (Bötticher 1840), puis dans la monumentale introduction de la première édition de La Tectonique des Hellènes, «Pour une philosophie de la forme tectonique» (Bötticher 1844), où la dimension "hellénique» s'efface et laisse l'ambition conceptuelle se déployer dans toute sa pureté anhistorique $^{8}$. Sur cet horizon strictement spéculatif, le système bötticherien est généralement décrit comme un idéalisme radical, distinguant «l'essence» (Wesen) invisible, d'un côté, et le «corps» (Körper), sa manifestation visible, de l'autre. Le passage de l'essence au corps s'opère par la mise en forme (Gestaltung) qui est elle-même double, associant une forme nucléaire (Kernform) et une forme artistique (Kunstform), sachant que la forme nucléaire est aussi désignée, à partir de la seconde édition de La Tectonique, comme une forme «opératoire " (Werkform). Ces concepts clés - l'essence, le corps, la forme nucléaire, la forme opératoire, la forme artistique, la tectonique - constituent le squelette intellectuel de toute la pensée du savant. Ils appellent donc quelques remarques explicatives.

Première remarque: on a souvent suggéré que l'abandon de la notion de forme nucléaire au profit de celle de forme opératoire signifiait le passage, chez Bötticher, d'une approche philosophique purement spéculative à un objectif éducatif à destination des architectes et des dessinateurs industriels (Jones 2007). Entre 1840 et 1852, date d'achèvement de la première édition de La Tectonique, s'est incontestablement formulée une méditation sur la manifestation des essences à travers l'art, dont la forme nucléaire est définie comme la charnière. En regard, entre 1872 et 1881, dates de publication de la seconde édition complètement remaniée du même ouvrage, l'accent est mis sur l'apprentissage méthodique d'un langage formel, ce qu'exprime le nouveau titre de l'introduction du livre: la «philosophie de la forme tectonique» y est devenue une «leçon» (Lehre) «des formes tectoniques» (Bötticher 1872), le passage au pluriel semblant incarner le renoncement à la pureté intérieure du concept de forme au profit d'une description pratique de ses différents avatars. Ce tournant est dicté chez Bötticher par des considérations stratégiques, afin de rendre son œuvre plus immédiatement lisible par un public d'architectes et d'archéologues et de consolider ainsi sa position au sein des institutions académiques berlinoises ${ }^{9}$. II est probable qu'il reflète également l'impact de la lecture de Semper, à partir de 1860. Cependant, l'idéalisme ontologique de départ n'est pas fondamentalement remis en cause: si la «forme opératoire» se meut effectivement dans le champ de la stricte matérialité, elle n'en est pas 
moins toujours - et non sans difficultés dans le raisonnement - en contact avec l'«essence idéelle», qu'elle continue de devoir transcrire dans la réalité matérielle en tant que construction. À preuve, le fait que l'ancienne notion de forme nucléaire, avec ses connotations idéalistes, ne disparaît pas de la version tardive de La Tectonique, où elle est même nostalgiquement désignée, dans un passage de l'introduction, comme le terme sans doute le plus proche de la vérité métaphysique que Bötticher n'a jamais cessé de poursuivre:

Par forme opératoire, on désigne ce schéma constructif organique d'un constituant, schéma auquel incombe tout à fait exclusivement l'effort matériel et statique; c'est pourquoi on la nommait auparavant volontiers forme nucléaire du constituant, parce qu'elle en incarne le noyau statique fondateur ${ }^{\mathbf{1 0}}$. (Ibid. : 20)

Au total, Bötticher n'a jamais cessé de penser la tectonique comme une philosophie première de la construction, définie en tant que pratique matérielle commune à l'architecture et à tous les artisanats ${ }^{\mathbf{1 1}}$ et capable de se sublimer elle-même sur un plan idéel, en se fondant uniquement sur sa conceptualité propre, au-delà des finalités pratiques de l'objet construit proprement dit.

Deuxième remarque: la forme nucléaire (ou opératoire), quoique relevant du domaine de l'invisible, ne saurait être confondue avec l'idée ou l'essence idéelle. Au même titre que la forme artistique, elle constitue une étape dans un processus de concrétisation des essences, c'est-à-dire de descente de l'idée invisible dans la matière visible. C'est ce processus qui intéresse fondamentalement Bötticher et auquel il identifie la notion directrice de tectonique. Dans ce cadre, la forme nucléaire incarne la pensée constructive, la façon dont une idée de l'espace (Raumidee) va se concrétiser dans la définition d'un jeu de forces contradictoires de pesée et de poussée, de poids et de soutien, pour aboutir à un équilibre statique.

Troisième remarque: la forme artistique ne représente pas la forme nucléaire (ou opératoire) mais le processus tectonique dans son ensemble, lequel procède de l'association entre formes artistiques et formes nucléaires. Le fait que le processus formel artistique soit exactement «contemporain ${ }^{12}$ » du processus formel nucléaire est tout à fait essentiel. Forme nucléaire et forme artistique constituent comme l'avers et le revers d'une même médaille - inséparables et co-actives dans l'engendrement de l'œuvre tectonique:

Le principe hellénique qui consiste à rendre perceptible de façon imagée, sur le corps tectonique lui-même, par des formes artistiques analogiques, son action statique matérielle, de façon que, par la médiation de ces formes artistiques, le concept du corps considéré soit offert à la vision dans toutes ses relations, ce principe contient la seule loi valable en vertu de laquelle les créations tectoniques, surtout, peuvent être produites. [...] Ce principe a la même validité pour tous les produits de la tectonique, du plus petit outil jusqu'aux plus vastes constructions architecturales. (Ibid.: 27)
10. Je souligne.

11. "Chez Homère, le maître d'œuvre d'une habitation princière est un tectone au même titre que le sellier, le charron, le constructeur de machines, ou le cornier, le charpentier de navire, le menuisier et le forgeron. À l'origine, on ne trouve pas davantage de séparation entre l'invention et l'exécution, ou entre l'image artistique et le simple travail artisanal. » (Bötticher 1872: 4)

12. "Du point de vue de leur conception, les deux formes sont aussi contemporaines. " (Ibid. : 40) 
13. Alois Riegl, Stilfragen. Grundlegungen zu einer Geschichte der Ornamentik, Berlin, George Siemens, 1893. La grande différence avec la tectonique de Bötticher réside cependant dans le fait que ce renversement de la logique mimétique, chez Riegl, ne renvoie à aucun horizon ontologique.

\section{ci-contre \\ de haut en bas}

fig. 5

Karl Bötticher,

La Tectonique des Hellènes. Potsdam, Ferdinand Riegel, 1852, Atlas, pl. I. Photo (c) Universitätsbibliothek Heidelberg.

fig. 6

Karl Bötticher,

La Tectonique des Hellènes.

Potsdam, Ferdinand Riegel,

1852, Atlas, pl. III. Photo

(C) Universitätsbibliothek

Heidelberg.
C'est là la grande idée de Bötticher: la forme artistique ne vient pas prendre acte d'un système déjà constitué; elle le fait advenir dans l'acte même de sa représentation. Ce qui fait qu'elle se représente aussi nécessairement elle-même en représentant ce processus dont elle est un élément constitutif. Au moment - et au moment seulement - où s'opère cette autocompréhension artistique, la simple construction devient un système tectonique, c'est-à-dire la production consciente d'un «corps" matériel relié à une "essence» idéelle. La forme artistique fait ce qu'elle figure au moment où elle le figure. Pensée et production sont une seule et même chose: c'est parce que la tectonique se pense elle-même dans la forme artistique qu'elle se produit en tant que tectonique. Selon Bötticher, autrement dit, la tectonique est la construction parvenue, grâce à l'intervention de la forme artistique, au stade de l'autoreconnaissance, suivant une structure de mise en abyme à l'infini.

Quatrième remarque: ce processus d'autocompréhension accompli par la forme artistique est allégorique. Pour transformer une construction en organisme tectonique, la forme artistique en passe par des motifs naturels (la feuille d'acanthe ou la tige de roseau, par exemple). Mais ces motifs ne sont pas une fin en soi, ils ne sont pas justifiés par la mimésis. Pour Bötticher, à l'inverse de la doctrine vitruvienne, ce n'est pas la feuille d'acanthe qui a inspiré le chapiteau corinthien; c'est l'idée du chapiteau, c'est-à-dire de la rencontre entre un poids et une poussée, qui a inspiré le choix allégorique de la feuille d'acanthe pour se figurer elle-même (une direction de pensée que reprendra Alois Riegl dans ses Stilfragen ["Questions de style»] de $1893^{13}$, sans se référer explicitement à Bötticher). La forme artistique, constituée par des agencements de motifs végétaux ou animaux, est donc non seulement allégorique mais auto-allégorique. Elle allégorise l'avènement de la tectonique, dans laquelle elle est présente consubstantiellement. Un paragraphe fondamental "sur l'essence de l'analogie dans les formes artistiques tectoniques", dans la seconde édition de l'introduction de La Tectonique, le dit clairement; après avoir apparemment minoré les formes artistiques en les rejetant à l'extérieur du strict processus constructif, Bötticher en déduit que cela explique certes le recours à des motifs naturels (animaux ou végétaux), mais que, in fine, ce recours n'est pas mimétique:

Dans la mise en forme accomplie de chaque composante de la construction, il n'était emprunté à aucun modèle préexistant, quel qu'il soit, mais c'était sa propre image intellectuelle originaire, conçue en tant que pensée idéelle, qui était fidèlement imitée. Cependant, cette essence idéelle relevait de la forme opératoire uniquement, et pas des formes artistiques. Ces dernières n'y avaient accès que sur un mode allégorique, et en aucun cas par leur origine ou leur provenance, car elles procédaient, elles, de l'imitation de modèles déjà existants, tandis que la première avait conservé sa pure origine idéelle. Cette différence résidait dans les qualités exactement opposées des deux modalités de la forme, dont l'une accomplissait quelque chose d'effectivement matériel tandis que l'autre n'était qu'un élément explicatif de type allégorique. [...] D'où le fait que les formes artistiques, dans leur ensemble, ne pouvaient que se mouvoir dans la sphère des réminiscences d'objets de la perception, qui devaient évidemment déjà 

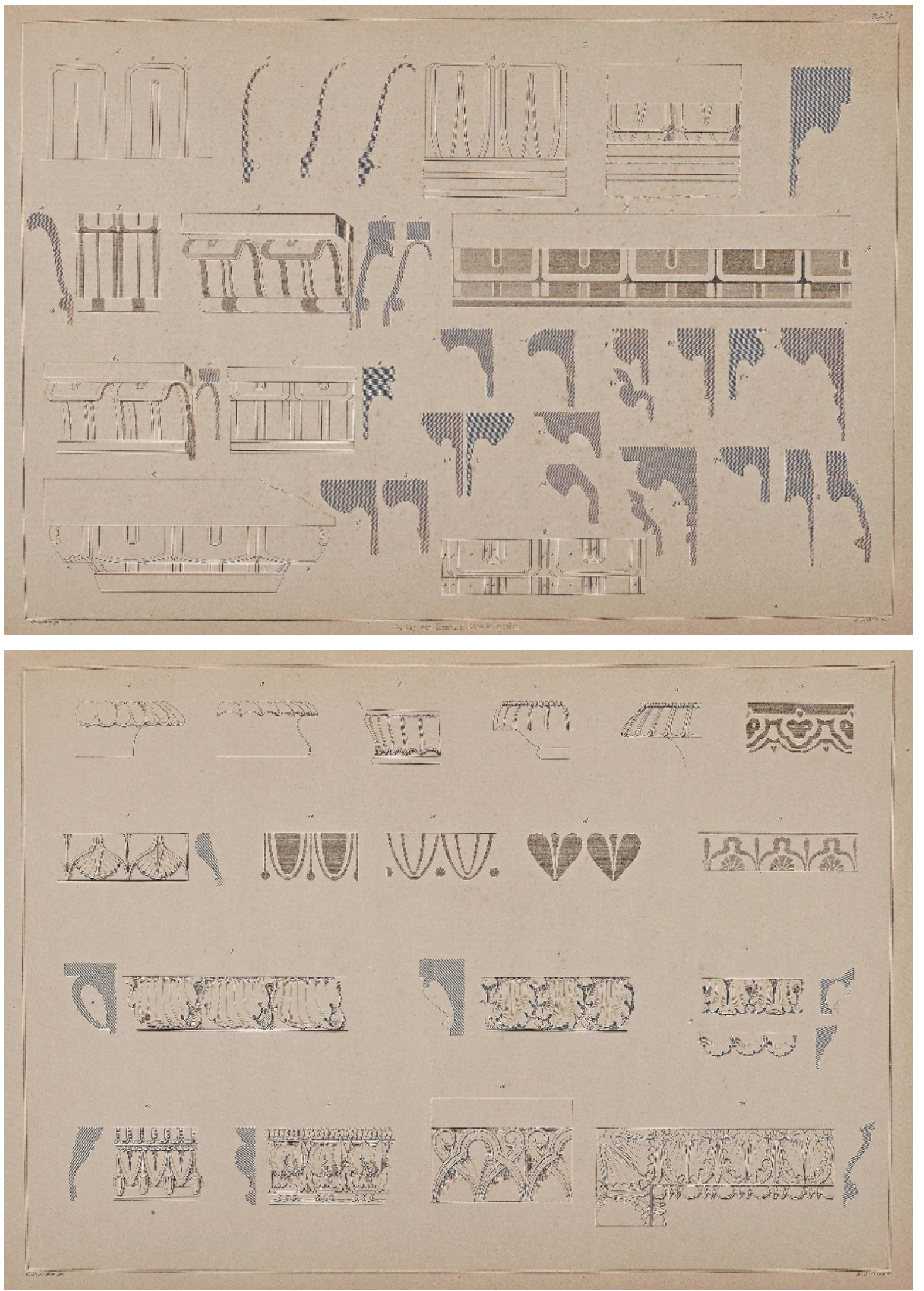
11.

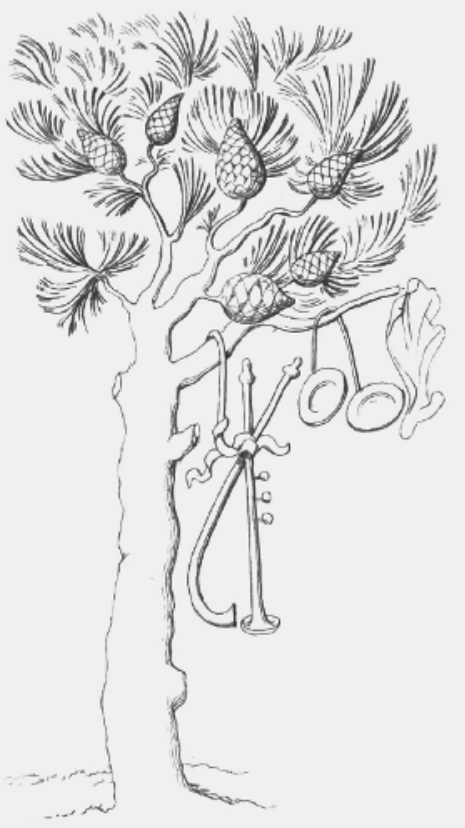

9.

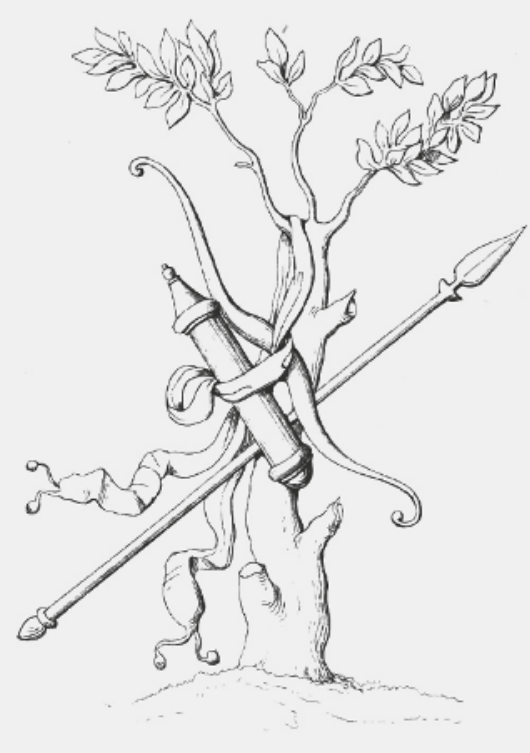

10.

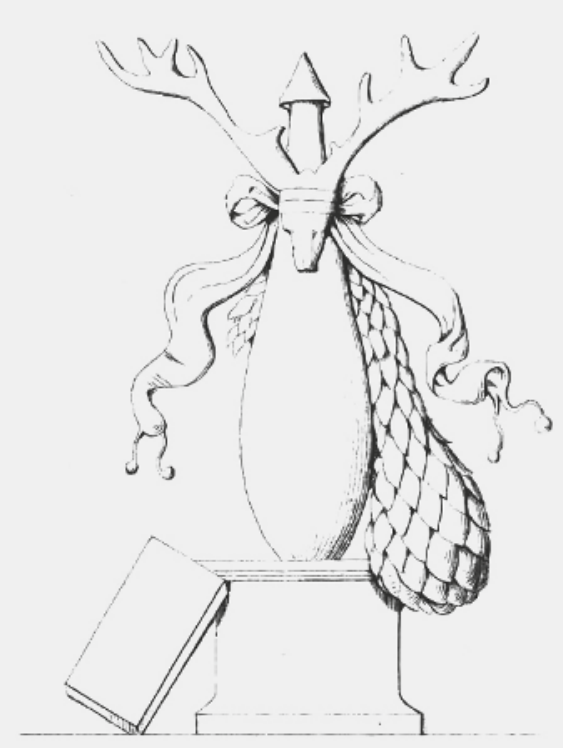


exister avant l'invention de la forme opératoire. Ainsi, les deux éléments de la forme se comportent, à n'en pas douter, de manière opposée. Cependant, tous les deux doivent contribuer comparablement à la manifestation figurale non seulement de la structure accomplie d'un bâtiment donné, mais aussi de l'articulation systématique entre tous ses constituants; tous deux, intimement unis, élaborent un seul et même concept tectonique ${ }^{14}$. (Bötticher 1872: 31)

Cinquième et dernière remarque: la forme artistique, en tant qu'elle pense et qu'elle produit simultanément un système tectonique, a un nom c'est l'ornement. De cette capacité d'intellection de la forme par elle-même, lui seul est porteur, à la différence de la peinture ou de la sculpture mimétiques. Non seulement il se singularise par là, mais surtout il porte à son sommet, ce faisant, la puissance créatrice de l'homme, intégrant dans un même mouvement la production et la pensée de la production, la création artistique et l'intellection de cette création ou, pour le dire plus trivialement, les forces de l'imagination et celles du raisonnement logique. C'est pourquoi, dans les planches de La Tectonique, c'est l'autonomisation du vocabulaire ornemental grec qui frappe surtout, détaché de son contexte tant architectural que mobilier, pour être strictement relié à une structure constructive interne (fig. 6). L'ornement y apparaît véritablement en tant que construction et non pas comme le compagnon servile de la construction. Il porte en lui la dynamique structurelle qu'il illustre.

L'ornement tectonique se présente donc comme une archi-forme, une forme princeps, inaugurale, en amont de l'architecture et des arts mineurs qu'il contribue à accomplir. C'est aussi une archi-pratique, dans la mesure où s'opère en lui l'union de la pensée et de la production: une production qui se pense en s'effectuant et une pensée qui se matérialise en se conceptualisant. De là vient sa supériorité sur les images mimétiques - ou «atectoniques» - de la sculpture et de la peinture, qui ne produisent ou ne modifient en rien la nature de ce qu'elles représentent (un tableau de paysage ne transforme pas le paysage qu'il dépeint). Enfin, l'ornement est une archi-langue, étant donné que sa structuration syntactique revêt en plus une dimension émotionnelle. En s'adressant à l'affectivité, en rendant en quelque sorte la logique des figures vivante et charnelle, elle produit une clarté intelligible qui passe par le corps et qui ne réclame donc aucun savoir préalable, bref qui est en mesure de faire coaguler tout un peuple dans une «compréhension originaire» (ibid.: 37) immédiate:

Il y avait dans les formes artistiques une langue d'images, qui n'était pas seulement comprise dans le cercle étroit des amateurs d'art, mais par le peuple entier, de la masse duquel étaient issus les tectones, à l'égal de la langue maternelle. (Ibid.)

Cette vision, on le sent bien, est sous-tendue par des préoccupations propres à l'Allemagne industrielle contemporaine. Chez Bötticher, l'éloge constant du peuple, qu'explique le souvenir fier et douloureux à la fois de ses origines sociales, est fondamentalement antidémocratique, hanté par la volonté d'opposer à la menace d'une modernité cosmopolite et polymorphe une intangible unité organique germano-hellénique.
14. Je souligne. 
15. Voir également Mayer 2004: 92-98
Au terme de ces remarques, une première conclusion s'impose: les sous-entendus politiques, les erreurs archéologiques ou l'obscurité conceptuelle des analyses bötticheriennes n'enlèvent rien à l'extraordinaire avancée conceptuelle que constitue l'hypothèse de la nature allégorique de l'ornement, à l'égard du principe constructif en tant que tel. Sa véracité historique discutable et ses déterminants idéologiques réactionnaires importent moins que sa productivité intellectuelle spécifique, pour la pensée de l'ornement dans l'Europe industrielle.

D'abord, elle met fin à l'opposition classique, winckelmannienne, qui consistait à distinguer ornement et allégorie au profit de la seconde, dans la mesure où l'ornement, selon Winckelmann, disjoignait forme et signification tandis que l'allégorie les conjoignait. L'ornement n'est plus un ajout, une addition facultative et superfétatoire à une signification déjà existante; il est au contraire projeté au sommet de la hiérarchie des formes, charnière opératoire entre le visible et l'invisible. Grâce à lui, une pratique mécanique de la construction se trouve fièrement enracinée dans une structure de l'être, douée d'un sens cosmologique. Cela situe évidemment le rapport de Bötticher avec la Grèce très loin de tout historicisme et lui permet de se faire le fervent défenseur de l'architecture en fer et des nouvelles formes qu'elle ne devait pas manquer de faire advenir, en accord avec une idée tectonique qui venait de Grèce, certes, mais qui ne nécessitait pas d'imiter superficiellement quelque «style» hellénique que ce soit (Bötticher 1846).

Ensuite, l'ornement est souverain dans l'édification de son propre concept: en pensant la tectonique, il se pense lui-même; la pensée de l'ornement émane de l'ornement même (ce dont Bötticher estimait être l'exemple vivant, lui qui avait gagné sa légitimité de théoricien en l'enracinant dans sa compétence première d'ornemaniste). Ce couronnement autoréférentiel est ce qui permet, cette fois, de dépasser les dogmes du fonctionnalisme et de projeter la création ornementale dans la sphère poḯtique, au sens aristotélicien du terme.

Enfin, comme pour toute construction métaphysique, ce qui caractérise La Tectonique de Bötticher, c'est son extrême cohérence interne, sa vision unitaire, derrière l'apparent dualisme de l'essence et du corps, de la forme nucléaire et de la forme artistique. Une chaîne continue d'engendrement logique du visible et des pratiques humaines se déploie à partir d'une "essence» invisible, puis remonte - par la voie de la représentation allégorique - de la technique la plus concrète à une idéalité ontologique fondatrice. À partir de là, les questions du style ou du système politico-social les plus adaptés à la modernité industrielle devenaient secondaires, alors qu'elles hantaient les débats intellectuels, particulièrement en Allemagne, depuis le début du siècle.

\section{Métaphysique et anthropologie}

Comme l'ont montré, entre autres, Wolfgang Herrmann (1981) et Harry Francis Mallgrave (1996: $219-225)^{\mathbf{1 5}}$, tout indique que cette cohérence spéculative, à l'écart de l'actualité, a fasciné Gottfried Semper, lorsqu'il a découvert la première édition de La Tectonique, à Londres, en 1852, dans 
la bibliothèque du British Museum. Un certain nombre d'axes directeurs de la pensée de Bötticher se retrouvent en effet dans les premières préoccupations de Semper, même si ce dernier les a rapidement dotés d'un sens différent: le philhellénisme bien sûr, se démarquant de l'héritage de Winckelmann pour réinterpréter Vitruve (fig. 8 et 9); la continuité entre théorie de la forme, enquête archéologique et réflexion anthropologique sur les usages de l'architecture; l'attention accordée aux procédures techniques les plus concrètes, porteuses de sens au sein d'une philosophie de la construction qu'incarne, dans les deux cas, le mot même de «tectonique»; enfin et surtout, la recherche d'une pensée de l'architecture dans et par l'architecture et l'identification, dans ce cadre, de l'ornement comme point focal de la réflexion.

Ce dernier axe est essentiel. Ce qui, chez Bötticher, constitue le fondement même du concept de tectonique, à savoir la présence de l'ornement en tant que figure simultanément constructive et autoréférentielle, a constitué un bouleversement majeur pour Semper, qui a montré le fruit qu'il en avait tiré dans une conférence sur les «symboles architecturaux» prononcée en janvier 1856 à l'hôtel de ville de Zurich sous le titre «De la conformité de l'ornement à des lois formelles et de sa signification comme symbole de l'art » (Semper 1856: 101-130). Là se trouve affirmée l'idée spécifiquement böttichérienne que l'ornement est le pivot de toute pensée de la forme. Même si, à la différence de Bötticher, Semper ne développe pas l'hypothèse d'une union spéculative entre forme artistique et forme nucléaire - et donc de l'ornement en tant que structure allégorique autoréflexive -, il appuie son raisonnement sur une ontologie de la forme, qu'exprime la notion grecque de cosmos, entendue dans son double sens d'harmonie universelle (le cosmique) et de parure (la cosmétique):

La riche et précise langue des Hellènes a le même mot pour désigner l'ornement dont nous nous parons nous-mêmes, ainsi que nos objets de prédilection, et la légalité suprême de la nature, l'ordre du monde.

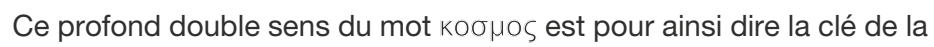
conception hellénique du monde et de l'art. Pour l'Hellène, la parure, dans sa légalité cosmique, était le reflet de l'ordre général du monde, tel que nous pouvons l'appréhender par les sens dans le monde des phénomènes. II le considérait comme un symbole de la légalité naturelle universellement compréhensible et fournissant lui-même son explication, y compris dans l'art plastique, qui apparaît partout comme un élément essentiel d'agencement formel, notamment dans l'art cosmique par excellence, l'architecture. (Ibid.: 101)

Pour Semper comme pour Bötticher, la «légalité» des diverses catégories d'ornement, c'est-à-dire leur accord avec les lois du cosmos, en fait les messagères immédiates, dans la vie quotidienne du peuple, d'une structure idéale de l'être. Du reste, un peu plus tard, en 1860, la première figure du Style - des cristaux de glace géométrisés - se rapproche encore davantage de l'idéalisme de Bötticher et de ses disciples (comme Johann Eduard Jacobsthal, dans sa Grammatik der Ornamente [“Grammaire de l'ornement »] 1874 [fig. 11] ) que de l'éclectisme ethnographique d'Owen Jones, dont la propre Grammar of Ornament (1856: 14) s'ouvre, quant à elle, avec pour 
16. L'opposition entre approche ontologique et approche anthropologique repose ici sur un emplo étymologique des deux termes. L'ontologie est prise en son sens strict de discours de vérité sur l'être; l'anthropologie, parallèlement, en son sens de discours de vérité sur l'homme; et l'une et l'autre sont saisies dans le seul cadre historique de la vision occidentale du monde. D'autres définitions seraient possibles: pour Philippe Descola (2010), par exemple, les ontologies sont définies en tant que visions du monde, considérées dans leur diversité fondamentale au sein des cultures humaines de toutes origines. Selon cette définition, une opposition entre horizon ontologique et horizon anthropologique n'aurait aucun sens, puisque les ontologies sont les objets que l'anthropologie se donne pour tâche de comprendre.

17. Voir sur cette question Papapetros 2013a: 1 et 6 .

\section{ci-contre}

fig. 8

Karl Bötticher, La

Tectonique des Hellènes.

Berlin, Ernst \& Korn

(Gropius'sche Buch-und

Kunsthandlung), $2^{\mathrm{e} e ́ d .}$

Atlas, 1873, pl.XVII. Photo

(C) Universitätsbibliothek

Heidelberg. première illustration, avant les motifs géométriques décontextualisés des planches proprement dites (fig. 10), un crâne tatoué de femme maorie (Varela Braga 2012: 2-18).

Sans jamais récuser explicitement cet horizon ontologique dont $L a T e c$ tonique de Bötticher lui avait offert un exemple monumental, Semper s'en est cependant détourné pour donner la primauté, dans le développement de sa propre pensée, aux différentes façons dont l'homme habite le monde et lui donne forme: à une quête philosophique de la vérité de l'être, il a clairement préféré une enquête anthropologique sur les racines des pratiques humaines ${ }^{16}$. Dès 1856 , pour expliquer la création de formes ornementales, il a recours à la notion comportementale de «pulsion décorative ${ }^{\mathbf{1 7}}$ » (Semper 1856: 102), qu'il conforte quatre ans plus tard, dans les "Prolégomènes" du Style, par celle d'«instinct cosmogonique" (Semper 1860: XXI) - et non de cosmogonie en tant que telle. Plutôt qu'à la structure de l'être, il s'intéresse à ses modes de représentation et aux comportements et usages que ces représentations suscitent. Du coup, le concept de tectonique, qu'il s'approprie, se trouve vidé de la portée ontologique dont l'avaient doté les grands échafaudages métaphysiques de Bötticher.

Semper n'affronte pas directement son prédécesseur; il se contente de le contourner, en se référant plutôt, pour parler de tectonique, à l'archéologie pratique de Karl Otfried Müller. Ce n'est que vers la fin du premier volume du Style, dans le chapitre sur "les aspects techniques et historiques" de l'art textile, quand il aborde les décors de l'architecture grecque, qu'il formule explicitement sa remise en cause du concept böttichérien de tectonique et, plus particulièrement, de l'articulation entre forme nucléaire et forme artistique. Le procédé consistant à ne citer qu'au bout de près de quatre cent cinquante pages un auteur aussi majeur, à l'époque, que Bötticher, et aussi directement associé aux thèmes traités par Semper dans son propre ouvrage, est évidemment d'une extraordinaire et moqueuse désinvolture, laquelle s'accuse dans la substitution intentionnelle du mot «schéma» au mot «forme», pour opposer le nucléaire et l'artistique, comme si la précision des concepts incriminés n'avait aucune importance:

Le style de construction grec ne fait pas de différence entre le "schéma nucléaire» et le "schéma artistique " - une distinction dans laquelle se trouve immanquablement contenue une pensée servilement égyptisante. Monsieur le Professeur Karl Bötticher - avec tout le respect dû à son érudition, à son goût et à son acuité d'esprit était inspiré par Hermès Trismégiste (lequel guidait aussi l'esprit de Pythagore) lorsqu'il écrivait ses livres d'exégèse sur le temple hellénique. (Ibid.: : 444)

Cette critique intervient au terme du raisonnement suivant. Pour Semper, le style grec a deux antécédents: les décors chaldéo-assyriens, où les dimensions constructive et ornementale ne font qu'un; et les décors égyptiens pharaoniques, qui dissocient au contraire les deux, assumant ainsi leur «essence anti-structurelle» (ibid. : 443). Selon lui, les décors grecs procèdent de l'Égypte dans la mesure où ils ne constituent pas en euxmêmes des éléments de la structure constructive; cependant, ils expriment 


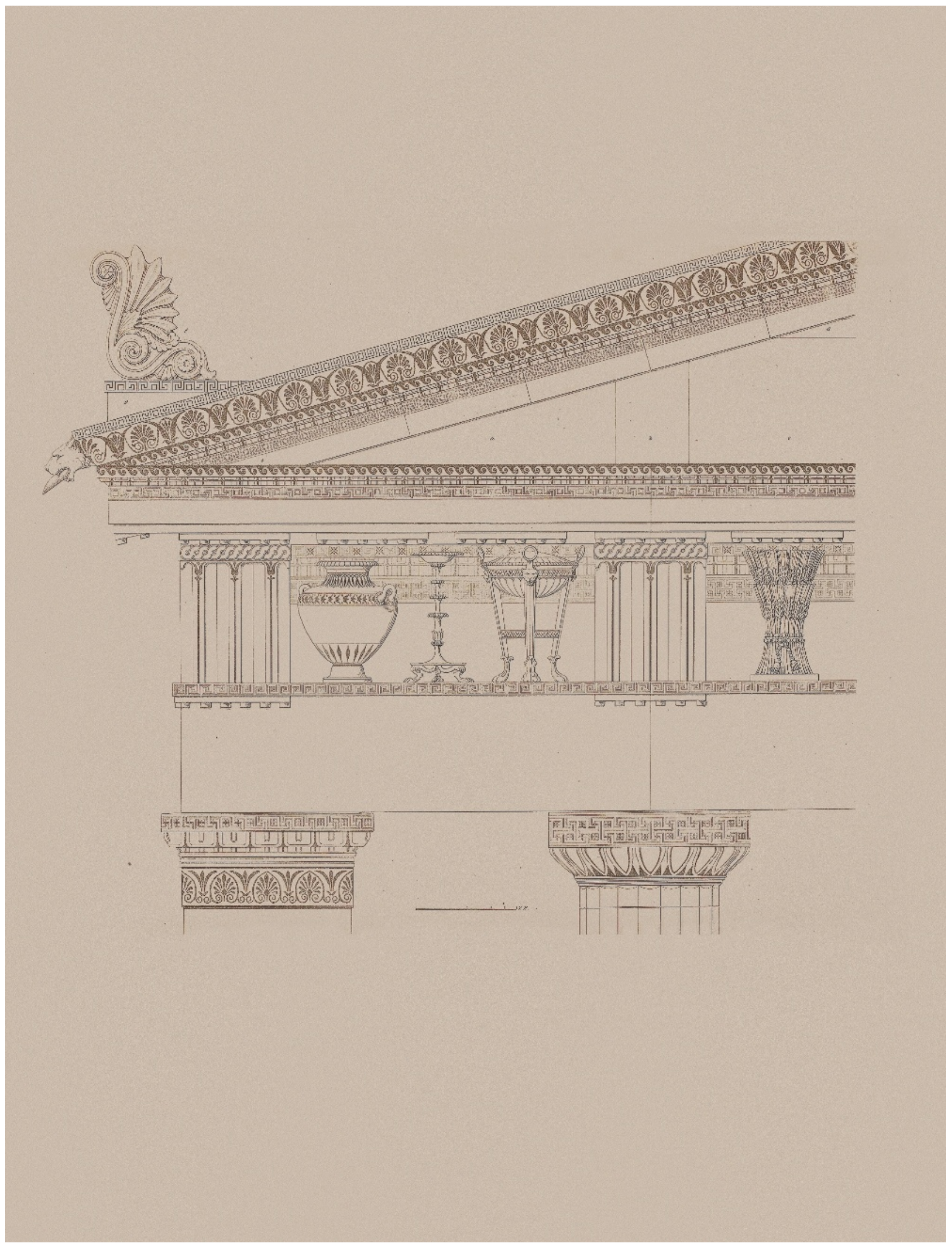




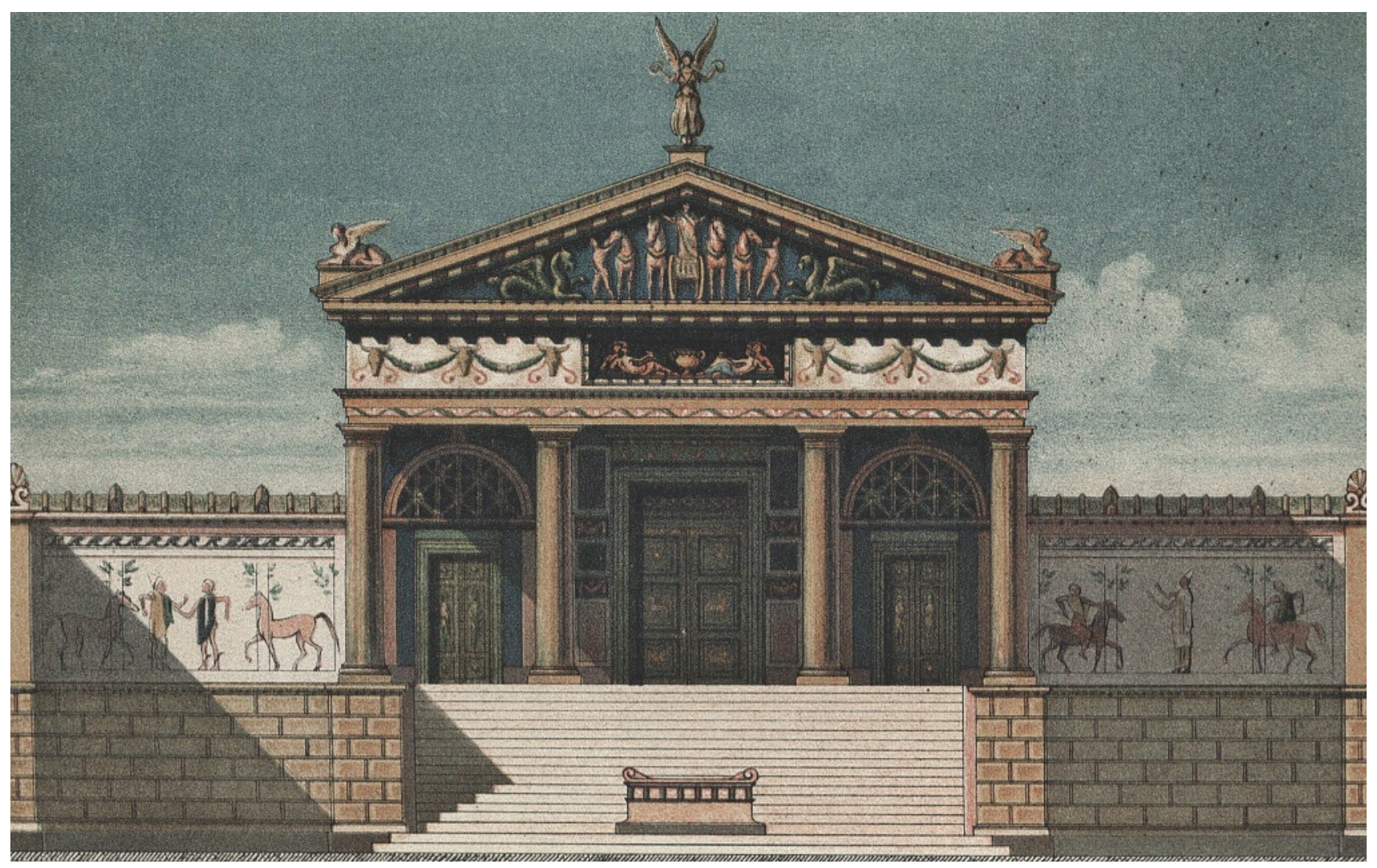

fig. 9

Gottfried Semper,

"Temple toscan d'après Vitruve", Le Style dans

les arts techniques et tectoniques..., Francfortsur-le-Main, Verlag für Kunst und Wissenschaft, 1860, t. I, pl. XIII.

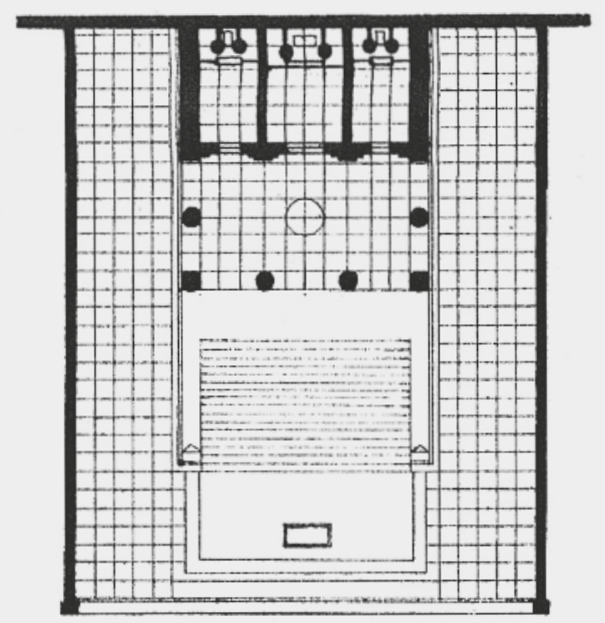

Toseanischer 'Tempel nach Vitruv. 
cette structure sur un plan strictement formel. Parvenu à ce stade de son raisonnement, Semper n'aurait pas eu de mal à le rattacher à l'articulation forme artistique/forme nucléaire chez Bötticher. Or, il fait exactement le contraire, en travestissant le sens de ces concepts clés. II laisse en effet supposer que l'auteur de La Tectonique (ou plutôt de toutes ces «exégèses" qu'il laisse volontairement innommées) a purement et simplement séparé forme nucléaire et forme artistique (alors que tout l'ouvrage est bâti sur la démonstration du lien entre les deux, condition pour qu'apparaisse une structure tectonique). Semper a alors beau jeu de cantonner la pertinence des catégories de Bötticher à la seule Égypte, là où le décor était autonome par rapport à la construction, et de se moquer de l'aveuglement du «professeur », qui aurait décrit des conceptions égyptiennes lorsqu'il croyait parler de la Grèce classique (comme les Grecs perpétuaient des cultes à mystères égyptiens lorsqu'ils révéraient Hermès Trismégiste).

En amont de cette querelle généalogique, l'enjeu est celui du rapport entre signification des formes et matérialité. Pour Bötticher, le génie de l'ornement grec est d'allégoriser la matérialité de la construction et de la relier ainsi au ciel des idées. Pour Semper, au contraire, cette matérialité est étrangère à la signification de l'ornement grec. C'est l'évacuation du matériau constructif en tant qu'horizon symbolique du décor qui lui permet de mettre en avant la dimension organique de l'architecture. De même que la vie d'un organisme n'est pas égale à la somme des matériaux qui conditionnent pourtant son existence, de même le sens d'une forme architecturale grecque n'équivaut pas à la somme de ses propres matériaux:

La forme s'éclaire uniquement à partir d'elle-même et de l'idée organique qui repose en elle, comme celle des créatures vivantes, à propos desquelles on ne demande pas en quelle matière elles sont faites, alors que la qualité et la quantité de ce matériau conditionnent au plus haut point leur existence, qui se modifie en fonction d'elles. (Semper 1860: 444)

En d'autres termes, Semper substitue l'idée de vie à celle de vérité, alors que Bötticher cherche à asseoir la vérité ontologique ultime des formes sur la matérialité du corps architectural. Semper n'ignorait pas cet horizon ontologique de La Tectonique, lui qui en avait attentivement lu la première édition et y avait fait écho à l'ouverture de sa conférence de 1856. Mais sa position était compliquée par le fait qu'il ne pouvait ni l'assumer (puisque sa propre approche organiciste le contredisait) ni le renverser frontalement (car, on va le voir, il n'était pas prêt à faire complètement le deuil d'un discours de vérité métaphysique). D'où cette déconstruction par la bande, qui refoule Bötticher dans les marges et le rature, en quelque sorte, en rendant ses concepts à la fois méconnaissables et anecdotiques. Les deux dimensions nucléaire et artistique sont présentées comme totalement distinctes alors qu'elles sont totalement indissociables, et la tectonique, fruit de leur union, n'est même pas mentionnée à ce stade. Semper en profite pour dérober au passage la référence hellénique à Bötticher en n'accordant de pertinence à la pensée de ce dernier que pour l'Égypte. Et par là même, il l'immerge, ou plutôt l'engloutit dans la relativité de l'histoire, ignorant superbement sa prétention à incarner une vérité universelle et suprahistorique. 
18. Voir Thibault 2016 : 111-112. L'auteure synthétise et analyse les nombreuses études qui, depuis un quart de siècle, ont renouvelé en profondeur la compréhension de l'analogie textile chez Semper, de Harry Francis Mallgrave à Michael Gnehm et, actuellement, à Sonja Hildebrand et Philip Ursprung.
En retour, malgré la rencontre qu'on dit courtoise entre les deux hommes, à Venise en 1877, Bötticher s'est déchaîné, dans la seconde édition de son livre, contre la théorie "textile» de Semper. De fait, avant même de constater sa criante marginalisation dans l'ouvrage de Semper et les moqueries dont il finissait par être l'objet au détour d'un lointain chapitre, il n'a pas dû avoir de mal à comprendre, dès l'ouverture du Style, que la vérité tectonique y était dépossédée de sa matérialité spécifique - exactement à l'encontre de ce qu'il avait rêvé d'établir de son côté - par l'argument du textile comme point d'origine de l'architecture. Lui qui avait commencé sa carrière de dessinateur industriel en répertoriant des textiles médiévaux était d'autant plus à même d'apprécier les fondements et les enjeux de cette élection du textile comme matrice générale d'intellection des formes ornementales. Ce faisant, l'architecture n'était plus définie à partir de la construction proprement dite mais à partir d'une technique tout autre, dont l'ornement avait pour seule fonction de conserver la trace mémorielle:

Certaines des plus récentes omissions modernes vont plus loin encore que la fiction vitruvienne, jusqu'à se moquer du bon sens. Elles ne veulent rien savoir ni d'une relation statique entre les éléments d'un système constructif ou du corps des ustensiles, ni du fait que les formes artistiques tectoniques se réfèrent à cette relation sur un mode allégorique. Ces pensées cherchent plutôt l'origine du phénomène de l'œuvre d'art en son entier dans l'usage supposé de tissus multicolores et de motifs de l'«art textile», pour en faire découler ce qu'elles appellent le "style» dans l'architecture antique. (Bötticher 1872: 37)

En outre, les thèses de Semper touchaient un plan anthropologique que Bötticher avait lui-même investi en mettant en relation le développement des cultes et la construction des temples dans la Grèce archaïque. Pour ce dernier, les comportements religieux, techniques et artistiques des Grecs s'accordaient pour révéler et célébrer l'harmonie de l'être, en partant de la nature. En revanche, pour Semper, comme l'a mis en lumière Caroline Van Eck (2009), le paradigme textile reflète l'exigence pré-architecturale, atectonique, de ritualiser l'espace par «l'habillage» (Semper 1860: 217), c'est-à-dire un besoin anthropologique originaire de "masquer» la réalité (ibid.: 227-231), besoin qui constitue la définition la plus spécifique de l'œuvre d'art:

Je pense que l'habillage et le masquage sont aussi vieux que la civilisation humaine et que la joie qu'on éprouve à l'un et à l'autre est celle-là même qu'on éprouve à faire ce qui transforme les hommes en sculpteurs, en peintres, en architectes, en poètes, en musiciens, en dramaturges, bref, en artistes. (Ibid. : 227-231) ${ }^{18}$

De la question: "Pourquoi y a-t-il des ornements? ", dans La Tectonique, on passe à la question: "Que fait-on avec des ornements?", dans Le Style. Et ces deux questions ouvrent des horizons opposés: d'un côté, l'identité platonicienne entre le beau et le vrai, que Bötticher avait fait sienne; de l'autre, le désir de travestissement et la «joie» du grimage systématique de tout. Une anthropologie du désir de fictions spatiales et de rituels, identifié 
comme la source de l'esthétique et de la culture en général, se trouve ainsi substituée à une métaphysique de la vérité constructive. L'horizon ontologique et idéel, ce «noyau » invisible des essences si cher à Bötticher (même s'il a tardivement remplacé l'idée de forme nucléaire par celle de forme opératoire), se trouve en quelque sorte rendu à la terre et aux agissements des hommes par Semper. Ce qui était continu chez l'un, du ciel des idées aux entablements de pierre, pont jeté entre l'éther le plus épuré des essences et l'expérience la plus concrète du métier de constructeur, devient discontinu chez l'autre, dès lors qu'est repoussée dans les marges l'idée que l'ornement puisse incarner un discours sur l'être.

Cependant, l'opposition entre anthropologie sempérienne et ontologie böttichérienne est moins simple qu'il n'y paraît. Chez Bötticher, la question anthropologique est certes subordonnée au plan métaphysique: pour lui, incontestablement, les actions et les fictions humaines sont reliées avec plus ou moins de bonheur et de lucidité à un ordre ontologique que reflète la pure dynamique ornementale. Néanmoins, la forêt obscure de ces usages humains n'a jamais cessé de l'obséder. Dans ses travaux proprement archéologiques sur l'Erechthéion ou sur le temple d'Athéna Nikè sur l'Acropole d'Athènes, il tente inlassablement de restituer, comme à partir de traces fossiles, ce qu'il a cru être la vérité de la société grecque archaïque, en commençant par son rapport au sacré. Dans le quatrième tome de La Tectonique des Hellènes - «Le temple hellénique dans son organisation spatiale à des fins cultuelles » (Bötticher 1849 IV: 1-407) -, il se lance dans une vaste analyse d'anthropologie sociale et religieuse, en disséquant les liens entre les usages liturgiques et les formes architecturales du temple hellénique. Enfin et surtout, dans l'énorme ouvrage qui a suivi la première édition de La Tectonique, Der Baumkultus der Hellenen, nach den gottesdienstlichen Gebräuchen und den überlieferten Bildwerken dargestellt («Le Culte de l'arbre chez les Hellènes, décrit d'après les usages liturgiques et les images disponibles») [Bötticher 1856], il ambitionne de dégager les origines du polythéisme grec classique dans le culte animiste de ces «monuments de la nature» (Naturmale) que sont les arbres sacrés, anthropomorphisés par tout un appareil d'artificialia (donc d'ornements) qui articulent ainsi pour la première fois nature et culture et forment «les premiers signes figuraux et les premiers temples» (ibid.: 3), tant en Grèce (fig. 7) que dans les autres civilisations archaïques. Spyros Papapetros a montré la continuité entre ces analyses et «la résurgence des idées animistes» dans le discours anthropologique au XIX $x^{e}$ siècle, d'Edward Tylor à James Frazer (Papapetros 2013b). En somme, dans le labyrinthe des grands ouvrages de Bötticher, l'attirance pour une démarche anthropologique concurrence obstinément la foi métaphysique; le rêve de passer d'une archéologie matérielle à une archéologie des comportements contamine et trouble en profondeur la clarté cristalline de sa vision architecturale de l'être.

Réciproquement, chez Semper, l'approche anthropologique, quoique résolument mise au premier plan, ne parvient pas vraiment à opérer le travail de deuil que réclame l'abandon de la référence métaphysique originelle. De ce deuil inachevé, on pourrait déceler les symptômes dans les points aveugles du système, là où ce dernier semble se replier sur lui-même sans vouloir se connaître. C'est le cas de l'animisme latent qui sourd de loin en 
19. Il faut préciser que l'auteur interprète cette évolution empiriste comme un accomplissement du concept de tectonique, par opposition au "dogmatisme rigide" de Bötticher.

\section{ci-contre}

fig. 10

Owen Jones, «Ornements des tribus sauvages ", $L a$ Grammaire de l'ornement. Londres, Day \& Son, 1856 , chap. I, pl. I. loin dans Le Style, tel que l'ont repéré Caroline Van Eck (2009) et Spyros Papapetros (2013a): dans le mouvement même de la réflexion du savant, ou plutôt dans les replis de sa pensée, tout se passe comme si la frontière se brouillait de temps en temps, entre les exigences rationnelles de l'enquête culturelle et une adhésion affective à certaines résonances de son objet d'étude. La clarté du regard analytique s'entrelace à un obscur besoin de croyance; la rigoureuse mise en perspective critique des visions du monde et des pratiques humaines qui en émanent apparaît sous-tendue par une sorte d'inclination animiste au premier degré.

Au bout du compte, les longues analyses de structures technicomatérielles, qui dominent dans le second tome du Style (Semper 1863), se substituent aussi bien au relativisme critique de l'approche anthropologique qu'à la célébration fidéiste d'un socle métaphysique. Semper semble appeler cette démarche structurale à la rescousse pour stabiliser un dispositif intellectuel d'ensemble souterrainement menacé par ses contradictions intimes. S'ouvre alors le champ rassurant d'une «poétique de la construction", selon l'expression de Kenneth Frampton (1995), où la description des systèmes de tensions entre structures se déploie à l'infini. De son côté, Marco Pogacnik écrit:

II ne s'agit plus de la tectonique comme fondement mais comme technique matérielle de la Zimmerei, de la charpenterie, et de la syntaxe particulière qui en caractérise les formes élémentaires. La tectonique, pour Semper, n'achève pas la grammaire qui structure le langage de l'architecture, elle en constitue au contraire seulement une des articulations syntaxiques ${ }^{19}$. (Pogacnik 1999-2000: 18)

C'est dans ce sens structural que le terme de tectonique s'est diffusé dans la réflexion esthétique allemande à partir de la fin $\mathrm{du} \mathrm{XI}^{\mathrm{e}}$ siècle, en faisant référence à Semper davantage qu'à Bötticher (Schwarzer 1995: 171 sq; Koetz et Thibault 2012). La pensée d'une charnière entre l'idée pure et la matière constructive n'a pas été vraiment invalidée, mais elle a été négligée au profit d'une fascination un peu myope pour les principes fondamentaux du faire, pour la dynamique fabricatrice issue du jeu logique entre des structures stables, avec l'espoir que cette grammaire - matrice originelle du modernisme - se transforme naturellement en poétique.

\section{Coda}

Au fond, chez Bötticher et Semper comme chez nombre d'autres théoriciens de l'ornement à l'ère de l'industrie, l'identification de la forme ornementale en tant que moteur originaire de la culture a provoqué une instabilité fondamentale de la pensée, entre la mise au jour d'un ordre universel transcendant - ce dont rend compte la notion grecque d'ontologie - et l'analyse des ressorts immanents de la productivité humaine - suivant les procédures de la science anthropologique occidentale. Tout se passe comme si l'ornement avait été laissé maître d'un royaume vacant, à peine était-il sacré par les théories tectoniques en tant que forme princeps à partir de laquelle l'homme donne sens au monde. D'où, peut-être, sa marginalisation, à nouveau, dans la pensée occidentale, à partir du début $d u x^{e}$ siècle. Certes, le terme de 
WXYDE STANOME

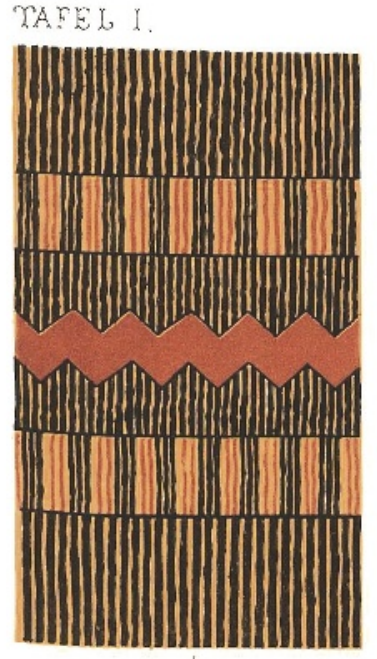

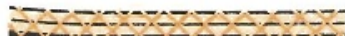

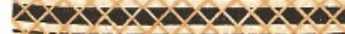

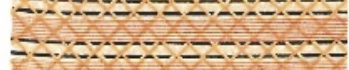

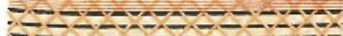
$1 \times<\times 8 \times<\times<\times<8$

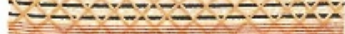
$x+x-2-2 x+x-5-2=$

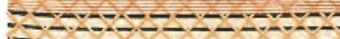

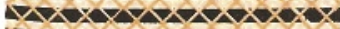

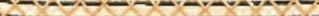

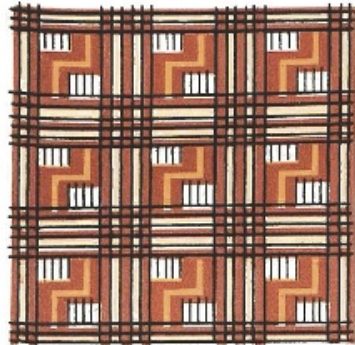

减 80808

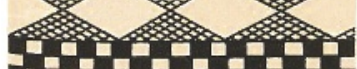

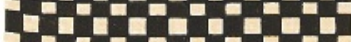
(6)

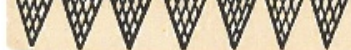

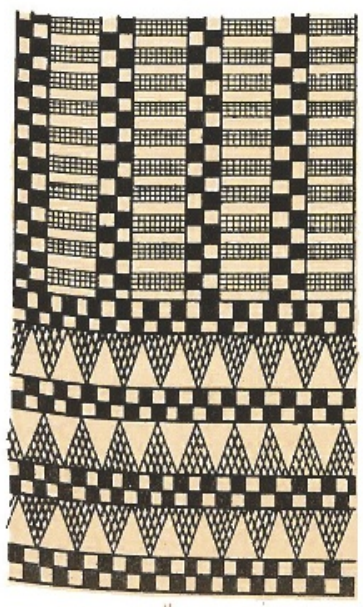

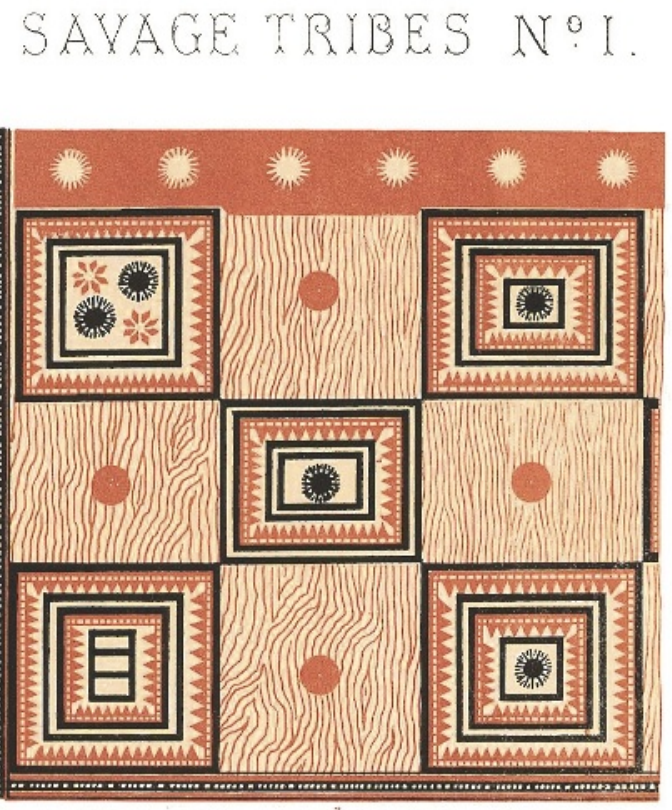
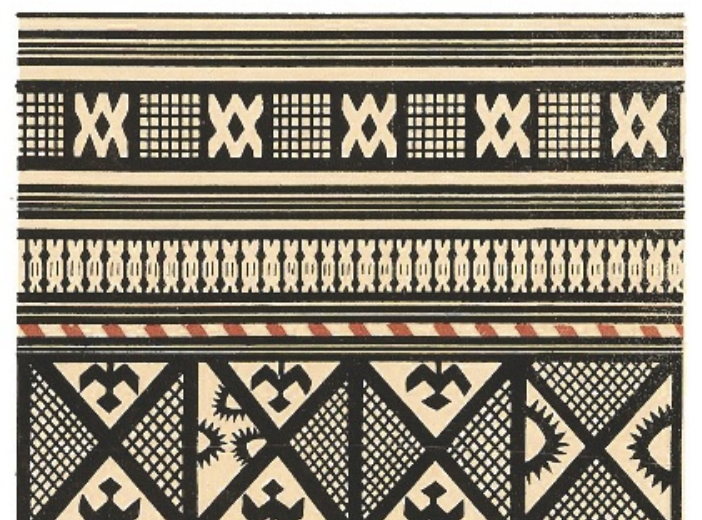
แนก W.
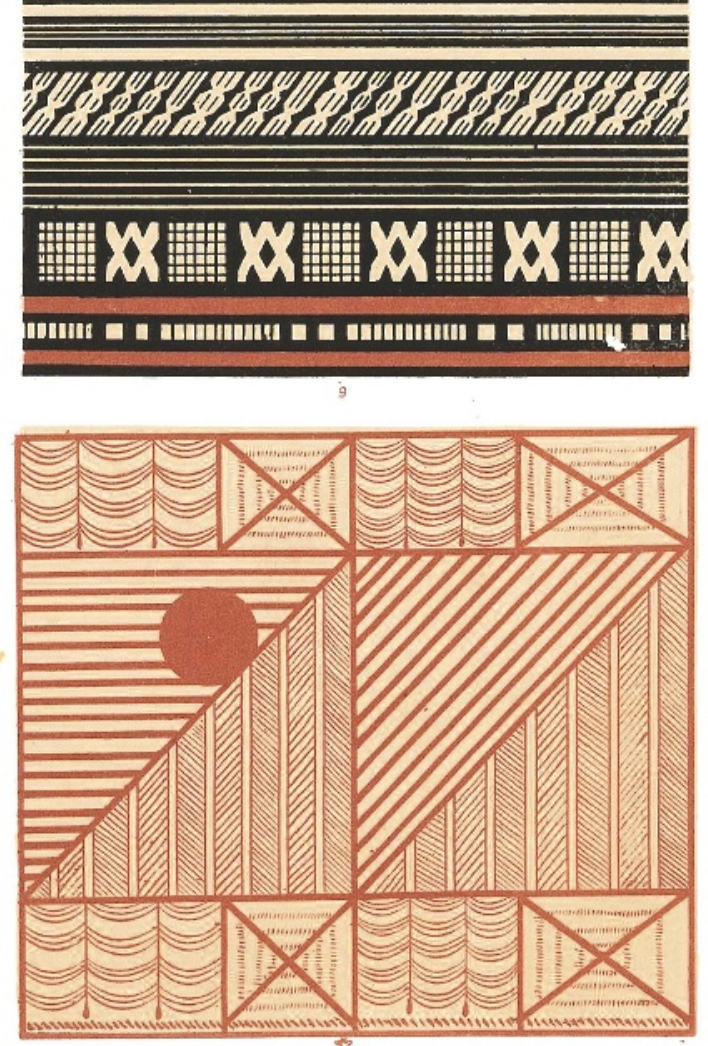
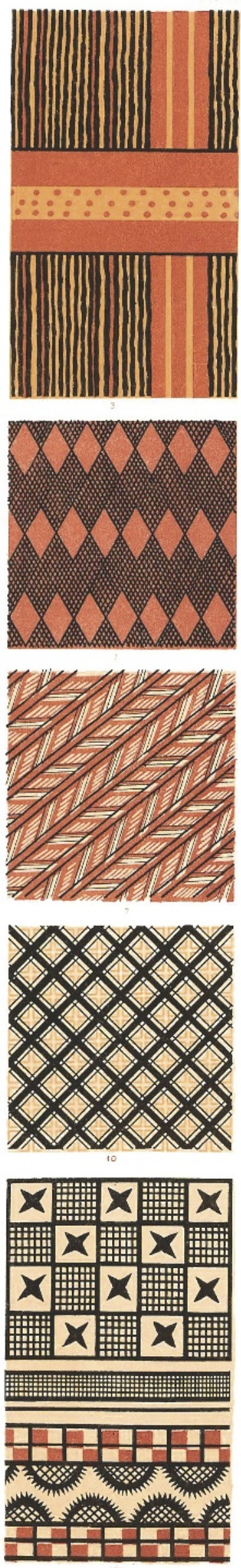


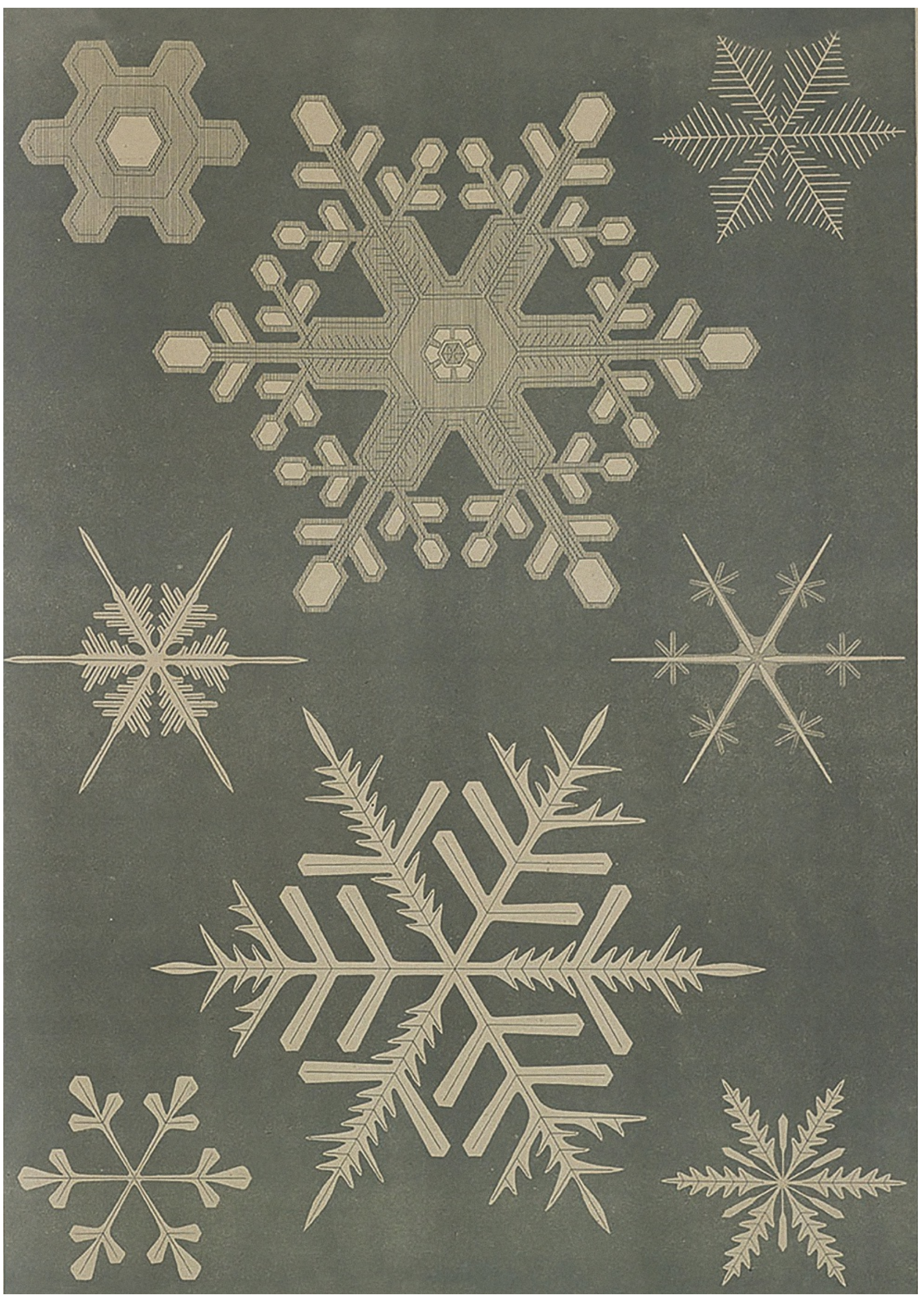


tectonique, grâce auquel ornement et construction s'étaient si puissamment confortés l'un l'autre, a continué à prospérer, notamment dans le domaine géologique, mais il s'est trouvé graduellement privé de son enracinement métaphysique comme de sa teneur anthropologique, au profit d'une pensée méthodique des structures directrices du faire, d'un mécanicisme généralisé.

On n'aurait donc affaire, avec les œuvres de Bötticher et de Semper, qu'à un pan d'histoire engloutie si n'y transparaissaient, à titre de symptômes, des besoins par lesquels continue de s'éclairer notre désir que le savoir critique propre à la modernité s'ajointe à quelque chose comme une croyance. L'hésitation, chez Semper, entre analyse rationnelle anthropologique et inclination affective animiste nous atteint ainsi sur un mode pathétique; et c'est ce que font aussi les lueurs de l'essentialisme métaphysique qui scintillent à l'horizon de la pensée de Bötticher. Ou pour le dire autrement: ce qui nous attache à ces frères ennemis en deçà de la lettre de leurs pensées, c'est notre inachevable travail de deuil à l'égard d'un horizon métaphysique qui, dans les formes construites, n'en finit pas d'échouer à se transformer en animisme. 
L'ornement à la conquête de soi. Tectonique, métaphysique et anthropologie chez Karl Bötticher et Gottfried Semper. Par Rémi Labrusse

\section{Beuth, Christian et Peter Wilhelm Friedrich (dir.)}

1821-1837 Vorbilder für Fabrikanten und Handwerker (t. I: Architektonische und andere Verzierungen; t. : II Geräthe, Gefässe und kleinere Monumente t. III : Vorbilder für die Verzierung von Zeugen und für die Würkerei insbesondere). Berlin, Petsch.

\section{Bötticher, Karl}

1840 «Entwickelung der Formen der hellenischen Tektonik", Wiener Allgemeine Bauzeitung 5 : 316-330.

1844 «Einleitung. Zur Philosophie der tektonischen Form ", in Die Tektonik der Hellenen. Potsdam, Ferdinand Riegel : 1-90.

1846 «Das Princip der hellenischen und germanischen Bauweise, hinsichtlich der Übertragung in die Bauweise unserer Tage ", Wiener Allgemeine Bauzeitung 11: 111-125.

1849 Die Tektonik der Hellenen, t. IV (Der Hellenische Tempel in seiner Raumanlage für Zwecke des Cultus). Potsdam, Ferdinand Riegel.

1856 Der Baumkultus der Hellenen nach den gottesdienstlichen Gebräuchen und den überlieferten Bildwerken dargestellt. Berlin, Weidmannsche Buchhandlung.

1872 «Einleitung. Zur Lehre der tektonischen Kunstformen ", in Die Tektonik der Hellenen, t. I (Die Lehre der tektonischen Kunstformen. Dorische, ionische und korinthische Bauweise). Berlin, Ernst \& Korn: 1-152.

\section{Demoule, Jean-Paul}

2014 Mais où sont passés les Indo-Européens?: le mythe d'origine de l'Occident. Paris, Seuil.

\section{Descola, Philippe}

2010 "Manières de voir, manières de figurer ", in La Fabrique des images: visions du monde et formes de la représentation, catalogue d'exposition (Paris, musée du quai Branly). Paris, Somogy/Musée du quai Branly: 11-18

\section{Ebe, Gustav}

1890 "Karl Boetticher als

Ornamentiker », Deutsche

Bauzeitung. Verkündigungsblatt des Verbandes deutschen Architektenund Ingenieur-Vereine 92: 553-554.

\section{Espagne, Michel}

2016 «Berlin-Athènes, MunichAthènes, Bonn-Athènes. Une géographie humboldtienne de l'Allemagne? ", in Michel Espagne et Sandrine Maufroy (dir.), L'Hellénisme de Wilhelm von Humboldt et ses prolongements européens. Athènes/Paris, École française d'Athènes/Demopolis : 97-118

\section{Espagne, Michel et} Maufroy, Sandrine (dir.)

2016 L'Hellénisme de Wilhelm von Humboldt et ses prolongements européens. Athènes/Paris, École française d'Athènes/Demopolis.

\section{Fittschen, Klaus}

2005 «Griechenland und der Orient: Ludwig Ross gegen Karl Otfried Müller », in Hans Rupprecht Goette et Olga Palaggia (dir.), Ludwig Ross und Griechenland. Akten des internationalen Kolloquiums. Rahden (Westphalie), Marie Leidorf : 251-260.

\section{Frampton, Kenneth}

1995 Studies in Tectonic Culture: The Poetics of Construction in Nineteenth and Twentieth Century Architecture, John Cava (éd.). Chicago/Cambridge (Mass.)/ Londres, Graham Foundation for Advanced Studies in the Fine Arts/The MIT Press.

\section{Herrmann, Wolfgang}

1981 "Semper und Bötticher », in Gottfried Semper theoretischer Nachlass an der ETH Zürich Katalog und Kommentare. Bâle/ Boston/Stuttgart, Birkhaüser : 26-40.

\section{Jacobsthal,}

\section{Johann Eduard}

1871-1874 Grammatik der Ornamente: nach den Grundsätzen von K. Boetticher's "Tektonik der Hellenen». Berlin, Winckelmann.

\section{Jones, Owen}

1856 "Ornaments of Savage Tribes », in The Grammar of Ornament. Londres, Day \& Son. : 13-17.

\section{Jones, Susan}

2007 "The Evolving Tectonics of Karl Bötticher: from Concept to Formalism ", in Tectonics. Making Meaning, conférence. Eindhoven, University of Technology, Department of Architecture, Building and Planning.

\section{Koetz, Laurent et Thibault, Estelle}

2012 «Ornement architectura et expression constructive: concepts d'hier et débats d'aujourd'hui ", Images Re-vues 10 [en ligne], disponible sur: https:// imagesrevues.revues.org/2386, consulté le 5 mars 2017.

\section{Mallgrave, Harry Francis}

1996 Gottfried Semper: Architect of the Nineteenth Century. New Haven/Londres, Yale University Press.

\section{Mayer, Hartmut}

2004 Die Tektonik der Hellenen. Kontext und Wirkung der Architekturtheorie von Karl Bötticher. Stuttgart, Axel Menges.

\section{Michaelis, Adolf}

1903 "Bötticher, Karl », Allgemeine Deutsche Biographie 47: 144-154.

\section{Müller, Karl Otfried}

1824 Geschichte hellenischer Stämme und Städte, t. II

(Die Dorier). Breslau, Josef Max.

1830 "Einleitung, A. Theoretische. 3. Eintheilung der Kunst ", in Handbuch der Archaelogie der Kunst ( "Manuel d'archéologie artistique»). Breslau, Josef Max: 1-23.

\section{Papapetros, Spyros \\ 2013a "Warburg, lecteur de Semper: ornement, parure et analogie cosmique ", Images Re-vues hors-série 4 [en ligne], disponible sur: http:// imagesrevues.revues.org/2862, consulté le 5 mars 2017.}

2013b " "L'âme est l'architecte de son propre corps". Animisme et tectonique entre la forêt ancienne et la ville moderne ", Intermédialités histoire et théorie des arts, des lettres et des techniques 22 [en ligne], disponible sur: https:// http://www.erudit.org/fr/revues/ im/2013-n22-n22/1024115ar/, consulté le 5 mars 2017.

\section{Pogacnik, Marco}

1999-2000 «La dissolution de la grande forme", Faces. Journal d'architectures 47: 14-23.

\section{Semper, Gottfried}

1856 Über die formelle Gesetzmässigkeit des Schmuckes und dessen Bedeutung als Kunstsymbol. Zurich, Meyer \& Zeller «De la conformité de l'ornement à des lois formelles et de sa signification comme symbole de 'art ", repris dans Du Style et de l'architecture: écrits 1834-1869, trad. de l'allemand par Jacques Soulillou et Nathalie Neumann, Marseille, Parenthèses, 2007 : 235-264).

1860 Der Stil in den technischen und tektonischen Künsten, oder, Praktische Aesthetics. Ein Handbuch für Techniker, Künstler und Kunstfreunde, t. I: die textile Kunst, für sich betrachtet und in Beziehung zur Baukunst. Francfort-sur-le-Main, Verlag für Kunst und Wissenschaft.

1863 Der Stil in den technischen und tektonischen Künsten, oder, Praktische Aesthetics. Ein Handbuch für Techniker Künstler und Kunstfreunde, t. II: Keramik, Tektonik, Stereotomie, Metallotechnik. Munich, Friedrich Bruckmann. 


\section{N 1.7.}

Streiter Richard

1896 Karl Böttichers " Tektonik

der Hellenen " als ästhetische

und kunstgeschichtliche Theorie:

Eine Kritik. Hambourg et Leipzig

Leopold Voss.

Schwarzer, Mitchell

1995 German Architectural Theory

and the Search for Modern Identity.

Cambridge/New York, Cambridge

University Press.

Thibault, Estelle

2016 «La confection des édifices

analogies textiles en architecture

aux XIX et $x \mid x^{e}$ siècles ", Perspective.

Actualité

Van Eck, Caroline

2009 «Figuration, Tectonics and

Animism in Semper's Der Stil ",

The Journal of Architecture (14) 3 :

Varela Braga, Ariane

2012 «Les enjeux de la préférence

pour les arts extraeuropéens

dans le discours sur l'ornement

en Grande-Bretagne au milieu du

xIX ${ }^{\ominus}$ siècle ", Images Re-vues 10

imagesrevues.revues.org/2141

consulté le 5 mars 2017.

2014 « Règles et principes.

L'enseignement du dessin

d'ornement en Angleterre à la fin

du XvIII" siècle", in Ralph Dekoninck

Caroline Heering et Michel Lefftz

(dir.), Questions d'ornements

$X V-X V I I I^{e}$ siècles. Turnhout, Brepols:

295-301. page 50 et ci-contre Détail d'un chapiteau copte. Musée National. Photo Hervé Champollion/akg-images.

250 th

3.

1.

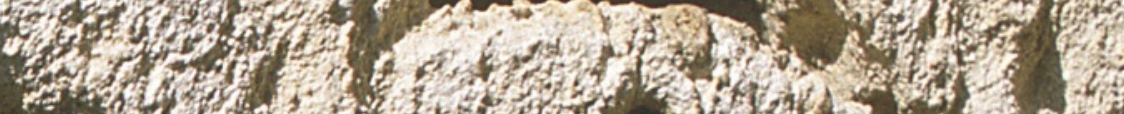

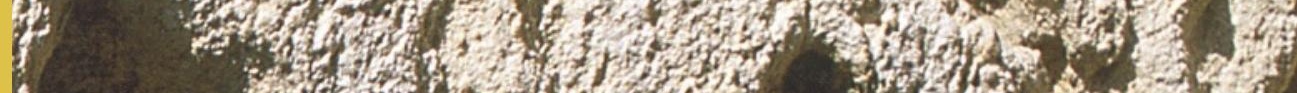

(4) 7.

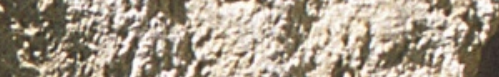

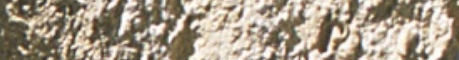

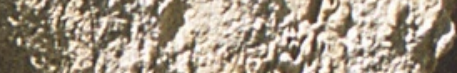

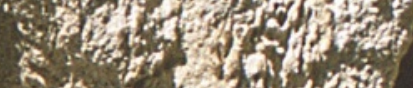

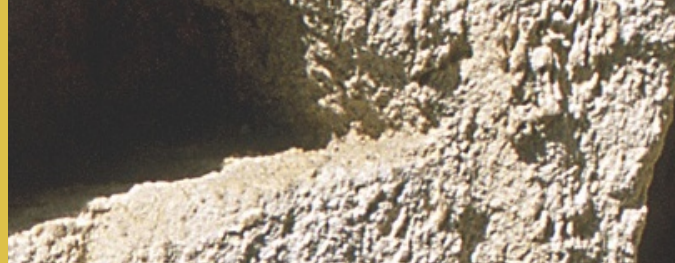

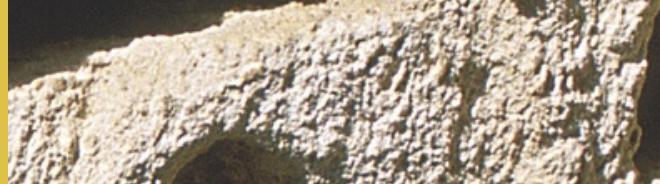

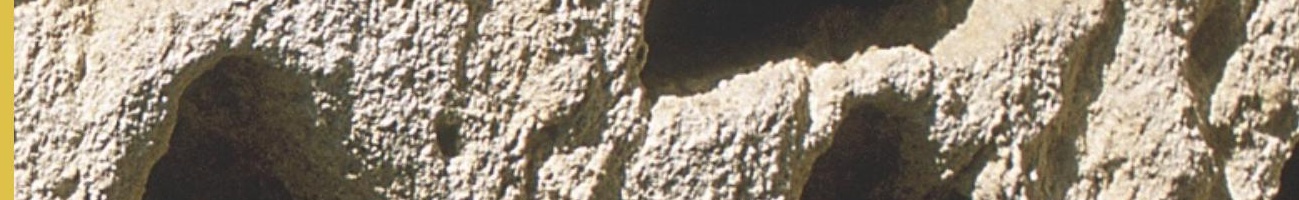

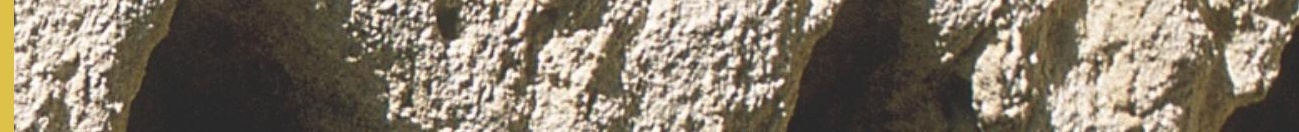

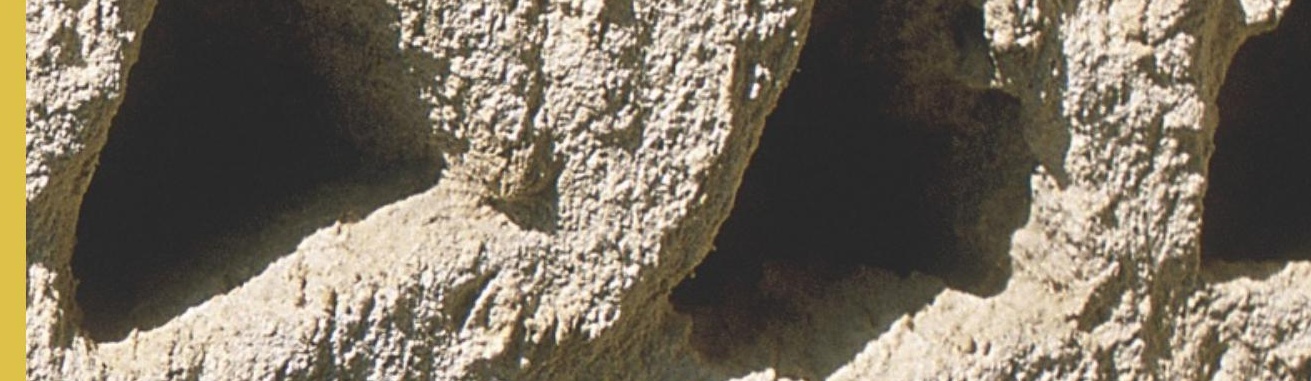
(n) W.

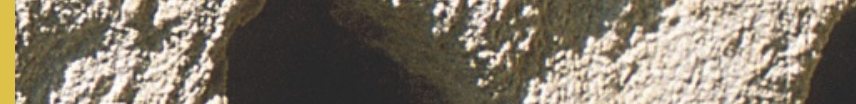

\section{tof \\ arition

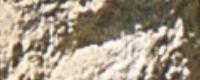

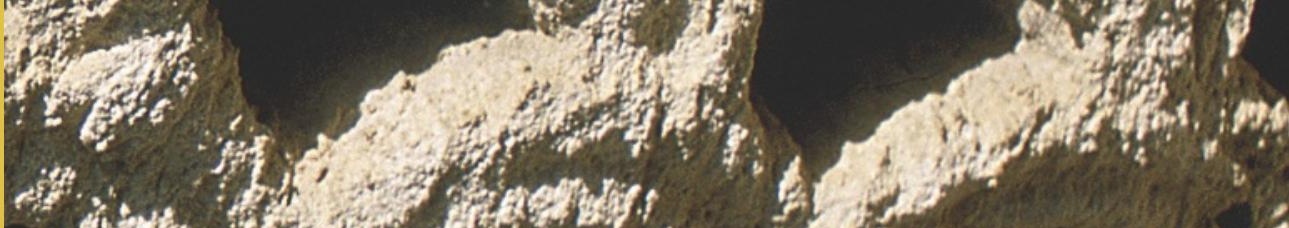

"

ton. 0

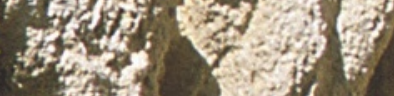

1.1

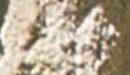

F.

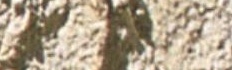

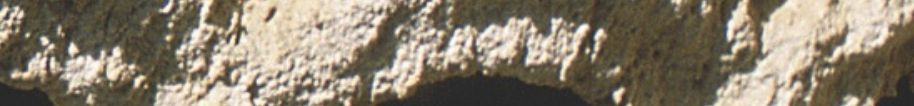

3 14010

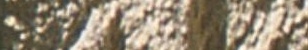

45 $x+4$ 2. IS 24

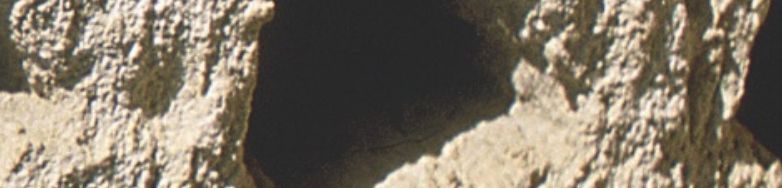

Q. 409
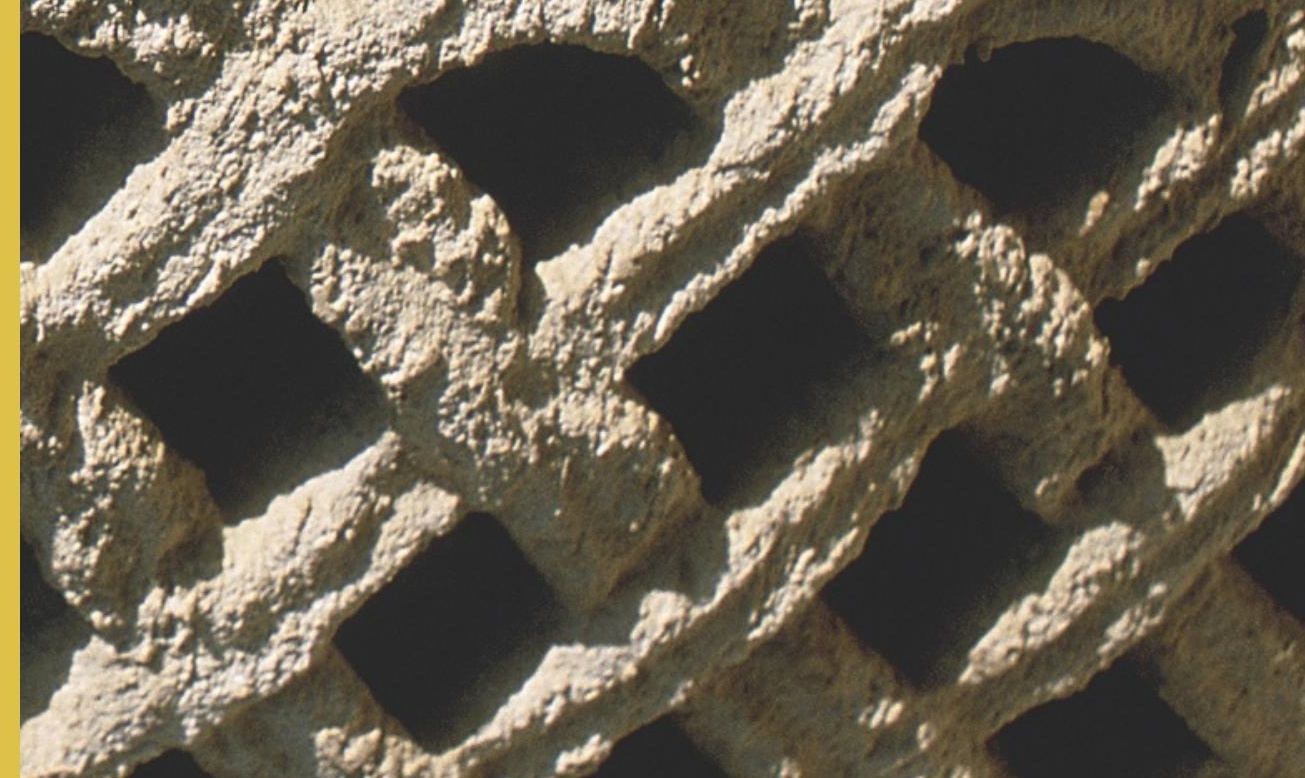

so

ef है

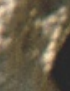

